\title{
Star formation histories in mergers: the spatially resolved properties of the early-stage merger luminous infrared galaxies IC 1623 and NGC 6090
}

\author{
C. Cortijo-Ferrero, ${ }^{1 \star}$ R. M. González Delgado, ${ }^{1 \star}$ E. Pérez, ${ }^{1}$ R. Cid Fernandes, ${ }^{2}$ \\ S. F. Sánchez, ${ }^{3}$ A. L. de Amorim, ${ }^{2}$ P. Di Matteo, ${ }^{4}$ R. García-Benito, ${ }^{1}$ \\ E. A. D. Lacerda, ${ }^{2}$ R. López Fernández ${ }^{1}$ and C. Tadhunter ${ }^{5}$ \\ ${ }^{1}$ Instituto de Astrofísica de Andalucía (CSIC), PO Box 3004, E-18080 Granada, Spain \\ ${ }^{2}$ Departamento de Física, Universidade Federal de Santa Catarina, PO Box 476, 88040-900 Florianópolis, SC, Brazil \\ ${ }^{3}$ Instituto de Astronomía, Universidad Nacional Autonóma de México, A.P. 70-264, 04510 México, Mexico \\ ${ }^{4}$ Observatoire de Paris, GEPI, Observatoire de Paris, PSL Research University, CNRS, Univ Paris Diderot, Sorbonne Paris Cité, \\ Place Jules Janssen, F-92195 Meudon, France \\ ${ }^{5}$ Department of Physics and Astronomy, University of Sheffield, Sheffield S3 7RH, UK
}

Accepted 2017 February 10. Received 2017 February 10; in original form 2016 July 28

\begin{abstract}
The role of major mergers in galaxy evolution is investigated through a detailed characterization of the stellar populations, ionized gas properties and star formation rates (SFR) in the earlystage merger luminous infrared galaxies (LIRGs) IC $1623 \mathrm{~W}$ and NGC 6090, by analysing optical integral field spectroscopy and high-resolution Hubble Space Telescope imaging. The spectra were processed with the STARLIGHT full spectral fitting code, and the emission lines measured in the residual spectra. The results are compared with non-interacting control spiral galaxies from the Calar Alto Legacy Integral Field Area survey. Merger-induced star formation is extended and recent, as revealed by the young ages (50-80 Myr) and high contributions to light of young stellar populations (50-90 per cent), in agreement with merger simulations in the literature. These early-stage mergers have positive central gradients of the stellar metallicity, with an average $\sim 0.6 \mathrm{Z} \odot$. Compared to non-interacting spirals, they have lower central nebular metallicity, and flatter profiles, in agreement with the gas inflow scenario. We find that they are dominated by star formation, although shock excitation cannot be discarded in some regions, where high velocity dispersion is found $\left(170-200 \mathrm{~km} \mathrm{~s}^{-1}\right)$. The average SFR in these earlystage mergers $\left(\sim 23-32 \mathrm{M}_{\odot} \mathrm{yr}^{-1}\right)$ is enhanced with respect to main-sequence Sbc galaxies by factors of $6-9$, slightly above the predictions from classical merger simulations, but still possible in about 15 per cent of major galaxy mergers, where U/LIRGs belong.
\end{abstract}

Key words: techniques: spectroscopic-galaxies: evolution-galaxies: interactionsgalaxies: ISM-galaxies: star formation-galaxies: stellar content.

\section{INTRODUCTION}

Merger-induced star formation was first predicted by Toomre \& Toomre (1972), and confirmed by Larson \& Tinsley (1978) in a study of optical colours of Arp galaxies. However, not all merging galaxies have significantly enhanced star formation rates (SFR). Recent results show that the enhancement of the star formation depends on multiple factors, such as the merger geometry and the properties of the progenitor galaxies (Di Matteo et al. 2007, 2008;

*E-mail: clara@iaa.es (CCF); rosa@iaa.es (RMGD)
Cox et al. 2008). For example, gas-rich $(S+S)$ merging pairs show significant enhancements in the specific star formation rates (sSFR) that are not found in S+E pairs (Cao et al. 2016). Moreover, a clear anticorrelation between the sSFR and the pair separation has been established. In particular, close pairs with projected separation $\leq 20 \mathrm{~h}^{-1} \mathrm{kpc}$ have an sSFR enhancement $\gtrsim 2$ (Ellison et al. 2008), while the sSFR enhancement of wider pairs is significantly less (Patton et al. 2013).

Besides enhanced SFR, the spatial extent of the star formation is an other important factor that must be taken into account when comparing the star formation in mergers with that in noninteracting control galaxies. Extended star formation is observed 
in many galaxy mergers from early-stage merger or separated progenitors (Wang et al. 2004; Elmegreen et al. 2006) to advanced or post-coalescence systems (Evans et al. 2008; Wilson et al. 2006). Recent high-resolution models determine the SFR efficiency in mergers by resolving parsec-scale physical processes (Teyssier, Chapon \& Bournaud 2010; Hopkins et al. 2013; Renaud, Bournaud \& Duc 2015). These simulations find that extended starbursts arise spontaneously during the first two pericentre passages, due to fragmentation of the gas clouds produced by an increase of the interstellar medium (ISM) supersonic turbulence as a consequence of the tidal interaction itself (Renaud et al. 2014). In these models, a merger-induced nuclear starburst is also present, but it starts later in the merger, during the final passage. They conclude that extended star formation is important in the early stages of the merger, while the nuclear starbursts will occur in advanced stages.

Mergers of gas-rich discs, such as those seen in luminous and ultra-luminous infrared galaxies (LIRGs and ULIRGs; Surace et al. 1998; Surace, Sanders \& Evans 2000; Veilleux, Kim \& Sanders 2002; Kim et al. 2013) are therefore extreme examples, where star formation is caught at its peak. They are unique laboratories offering insight into the physical processes triggered by galaxy mergers. The observational characterization of star formation in mergers at different stages is necessary to test the validity, and to put constraints on merger simulations.

To understand the role of major mergers in galaxy evolution, we have undertaken an integral field spectroscopy (IFS) study of galaxies in different stages across the merger sequence. In this paper, we focus on the detailed study of two LIRGs at an early merger stage: IC 1623 and NGC 6090. Both are classified as type IIIb systems according to the morphological classification of Veilleux et al. (2002). We aim to fully characterize their spatially resolved stellar population and ionized gas properties. In a future work, we plan to extend this study to merging systems observed in the Calar Alto Legacy Integral Field Area (CALIFA) survey (Sánchez et al. 2012), in order to cover different merger stages and to statistically study the evolution of merger-induced star formation.

The paper is organized as follows: Section 2 describes the observations and data reduction process. In Section 3, we summarize the results from the photometric analysis of the star clusters detected in Hubble Space Telescope (HST) images. In Section 4, we apply the fossil record method to analyse the stellar continuum and derive the spatially resolved stellar population properties: stellar mass and stellar mass surface density, $\mu_{\star}$; stellar extinction, $A_{V}$; luminosityweighted mean age, $\langle\log a g e\rangle_{\mathrm{L}}$; mass-weighted mean metallicity, $\langle\log Z\rangle_{\mathrm{M}}$; and the contributions to luminosity and mass of young, intermediate and old stellar populations (OSP). Section 5 presents results on the ionized gas emission, focusing on the morphology, nebular $A_{V}$, and the ionization conditions. We discuss the results in Section 6, and Section 7 presents the conclusions.

Throughout the paper, we assume a flat cosmology with $\Omega_{\mathrm{M}}$ $=0.272, \Omega_{\Lambda}=0.728$ and $H_{0}=70.4 \mathrm{~km} \mathrm{~s}^{-1} \mathrm{Mpc}^{-1}$ (Wilkinson Microwave Anisotropy Probe, 7-yr results). For IC 1623 redshift $(z=0.020067)$, this results in a distance of $86.8 \mathrm{Mpc}$. At this distance, 1 arcsec corresponds to $0.421 \mathrm{kpc}$. For NGC $6090(z=$ $0.029304)$, the distance is $127.7 \mathrm{Mpc}$, and 1 arcsec corresponds to $0.619 \mathrm{kpc}$.

\subsection{IC 1623}

IC 1623 (also commonly referred to as VV $114=$ Mrk 236; $L_{\mathrm{IR}}=4.5 \times 10^{11} \mathrm{~L}_{\odot}$; e.g. Sanders et al. 2003) is a pair of luminous infrared interacting galaxies at $86.8 \mathrm{Mpc}$, with a projected separation between the two nuclei of $6 \mathrm{kpc}$. The western progenitor, IC $1623 \mathrm{~W}$, is very bright in the UV and optical, with many star clusters detected in HST images. Its properties are very similar to Lyman break galaxies, and it is considered as the nearest Lyman break analogue (Grimes et al. 2006). In this paper, we are concentrating on the western progenitor, which is covered by our IFS data. The top left-hand panel of Fig. 1 shows the HST F435W image of IC 1623, with rectangles indicating the positions where we have obtained IFS observations. In contrast, IC $1623 \mathrm{E}$ is optically obscured but very infrared-bright. About half of the warmer dust traced in the mid-IR (MIR) is associated with the eastern galaxy, where both compact (nuclear region) and extended emission are found (Le Floc'h et al. 2002). Evidence for widespread star formation activity and shocks across the entire system is found in the UV, optical and MIR (Knop et al. 1994; Goldader et al. 2002; Rich, Kewley \& Dopita 2011). Analysing far-UV (FUV) and X-ray data, Grimes et al. (2006) found a galactic superwind in IC 1623 moving at $300-400 \mathrm{~km} \mathrm{~s}^{-1}$. Assuming a stellar origin for the hard X-ray emission (i.e. no contamination by an active galactic nuclei, AGN), they estimate a similar SFR for both progenitors: $\sim 28 \mathrm{M}_{\odot} \mathrm{yr}^{-1}$ for IC $1623 \mathrm{E}$ and $\sim 33 \mathrm{M}_{\odot} \mathrm{yr}^{-1}$ for IC $1623 \mathrm{~W}$. This is probably an upper limit in the case of IC $1623 \mathrm{E}$, as a highly obscured AGN was identified in sub-arcsec resolution ALMA observations of HCN (4-3) and $\mathrm{HCO}^{+}$(4-3) (Iono et al. 2013).

\subsection{NGC 6090}

NGC 6090 (Mrk $496=$ UGC 10267; $L_{\mathrm{IR}}=3.2 \times 10^{11} \mathrm{~L}_{\odot}$; e.g. Sanders et al. 2003) is another pair of luminous infrared interacting galaxies at $127.7 \mathrm{Mpc}$. The bottom left-hand panel of Fig. 1 shows the HST F435W image of NGC 6090. At optical and near-IR (NIR) wavelengths, NGC 6090 appears as a double nucleus system separated by $3.2 \mathrm{kpc}$ in projection (Dinshaw et al. 1999). The two galaxies are of roughly equal size, with a pair of tidal tails that have a full extent of $\sim 50 \mathrm{kpc}$. The north-eastern galaxy (NGC 6090 $\mathrm{NE}$ ) has a clear distorted spiral structure viewed face-on, while the south-western nucleus (NGC $6090 \mathrm{SW}$ ) is seen edge-on, with an amorphous morphology and no spiral arms visible. The most striking feature of NGC 6090 is the abundance of star-forming knots in the overlapping region, on the western side of NGC $6090 \mathrm{NE}$, and the paucity of similar clusters in its eastern spiral arms. Moreover, FUV and near-UV (NUV) spectroscopy shows that the NE nucleus is also dominated by a young starburst (González Delgado 2008). NGC 6090 SW also shows a similar abundance of blue knots on its eastern end, and one extremely luminous knot at the northern end. The molecular gas has a single component that peaks between the two galactic nuclei in the overlap region (Bryant \& Scoville 1999; Wang et al. 2004), suggesting that the gas responds to the interaction faster than the stars. Although the two galaxies show a strong $\mathrm{H} \alpha$ emission in their nuclei, the $\mathrm{H} \alpha$ emission appears to be brighter in the companion-facing side of each galaxy (Hattori et al. 2004). There is considerable evidence for starburst activity, but no evidence at optical or radio wavelengths for a compact AGN.

\section{OBSERVATIONS AND DATA REDUCTION}

\subsection{IFS observations}

The IFS observations we report here were taken using the Potsdam Multi-Aperture Spectrophotometer (PMAS) spectrograph (Roth et al. 2005) on the 3.5-m telescope of the Calar Alto Observatory (CAHA), with the Lens Array (LArr) configuration. We have 

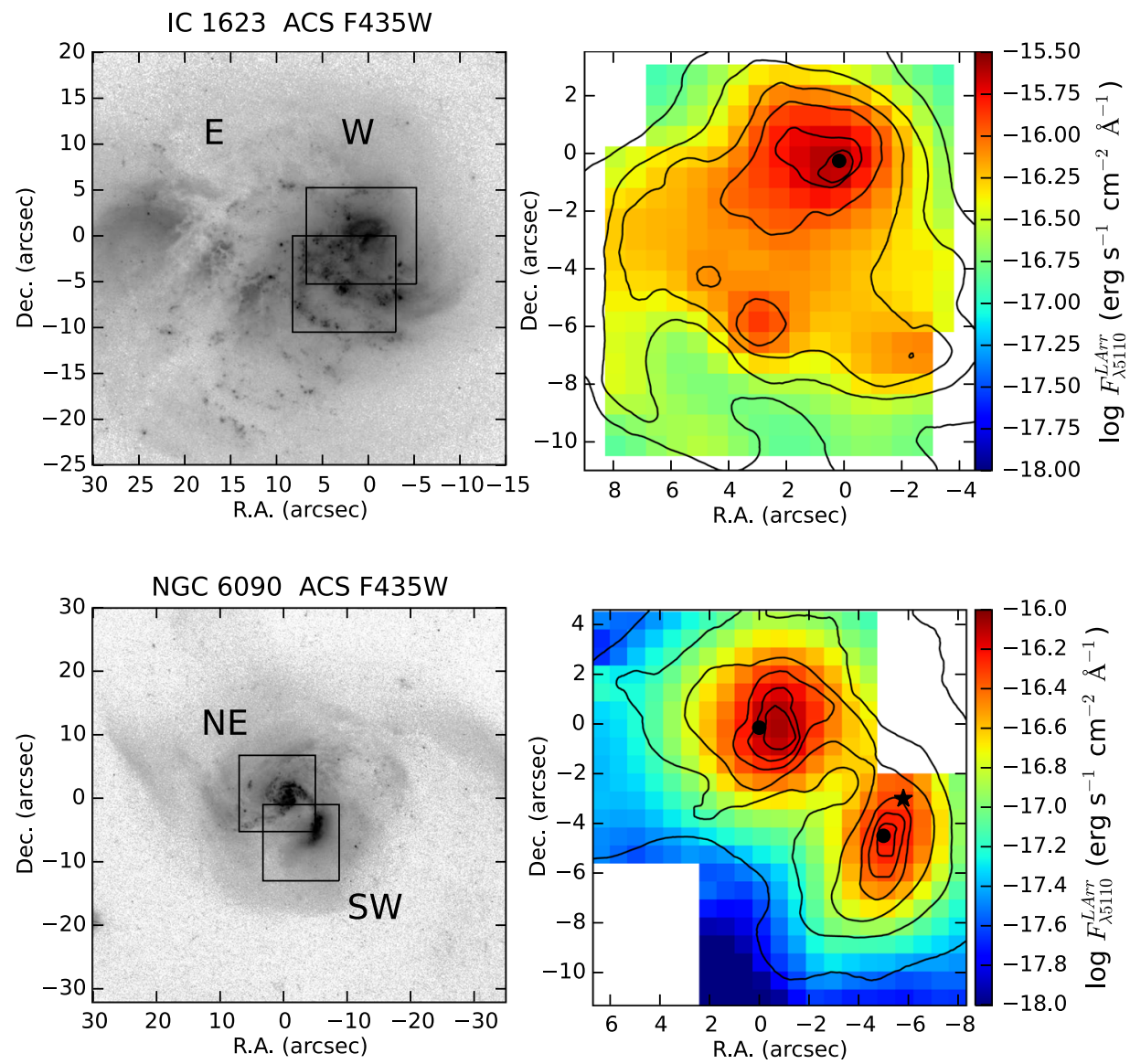

Figure 1. Observations of IC $1623 \mathrm{~W}$ (top panels) and NGC 6090 (bottom panels). Left-hand panel: HST ACS F435W images. The black rectangles indicate the positions where the IFS observations were obtained. Right-hand panel: PMAS LArr data continuum fluxes at 5110 Å rest-frame (obtained by averaging the continuum from 5050 to $5170 \AA$ ) presented on a logarithmic scale. Contours are from the HST ACS F435W image smoothed to the PMAS LArr resolution. 1 arcsec corresponds to $0.42 \mathrm{kpc}$ for IC 1623 and $0.62 \mathrm{kpc}$ for NGC 6090. The black circles indicate the expected position of the progenitor nuclei. In the case of NGC $6090 \mathrm{SW}$, the star indicates the position of the brightest knot.

used a spatial magnification of 0.75 arcsec lens ${ }^{-1}$, covering a 12 arcsec $\times 12$ arcsec field of view (FoV). From the standard stars and also the CAHA seeing monitor observations, we estimate that the seeing was in the range $\mathrm{FWHM}_{\mathrm{PSF}}=1.3-2.0$ arcsec for the observations. The V300 grating was used, providing a $3.2 \AA$ pixel $^{-1}$ dispersion and covering a wavelength range of 3700-7100 $\AA$ with a resolution of $7.1 \AA$ full width at half-maximum (FWHM, velocity resolution $\sigma \sim 170 \mathrm{~km} \mathrm{~s}^{-1}$ ). For each system, two pointings were taken, as shown in Fig. 1 by black rectangles over the HST images, with a total exposure time of 3 and 1.75 h for NGC 6090 and IC $1623 \mathrm{~W}$, respectively.

\subsection{IFS data reduction}

The PMAS LArr data reduction was performed using the $\mathrm{R} 3 \mathrm{D}$ package (Sánchez 2006), in combination with IRAF and our Own PYTHON scripts. The reduction consisted of the standard steps for fibre-based IFS, including bias subtraction, and a flat-field correction that was based on a master CCD flat provided by the CAHA. Calibration frames (continuum and $\mathrm{Hg} / \mathrm{Ne}$ lamps) were taken before the science frames to correct for the effects of flexure. The location of the spectra in the CCD was determined using a continuum-illuminated exposure. Each spectrum was extracted from the science frames by co-adding the flux within an aperture of 5 pixels $(3.75$ arcsec $)$ around this location along the cross-dispersion axis for each pixel in the dispersion axis. Wavelength calibration was performed using the $\mathrm{Hg}$ and $\mathrm{Ne}$ lamp exposures. The final accuracy of the wavelength calibration is $\sim 0.38 \AA$. Differences in the fibre-to-fibre transmission throughput were corrected by comparing the wavelength-calibrated science frames with twilight-sky exposures. The spectra were then flux-calibrated using the spectrophotometric standard stars Feige34 and BD+28D4211, observed in the same night. We corrected the standard stars data cubes from differential atmospheric refraction (DAR; Filippenko 1982). The median sky spectrum was obtained from the sky background frames, and subtracted from the target spectra. Once we had flux-calibrated and sky-subtracted all the individual frames for the same pointing position, they were combined and cosmic rays were rejected using IRAF's IMCOMBINE task. Data cubes were created, corrected from DAR, and rotated in a way that the north points upwards and the east leftwards. The two exposures at different locations were joined together in a mosaic.

Finally, we flux-recalibrated our LArr data using broad-band imaging from HST WFC435W in the case of IC 1623, and HST WFC435W together with SDSS $g$ and $r$ filters for NGC 6090. From the deviation between the photometry obtained from broadband imaging (in a 4.5-arcsec aperture) and the photometry extracted from the IFS in the same aperture, we found, in both systems, an average accuracy of the spectrophotometric calibration of $\sim 15$ per cent across the wavelength range covered by our data set. 

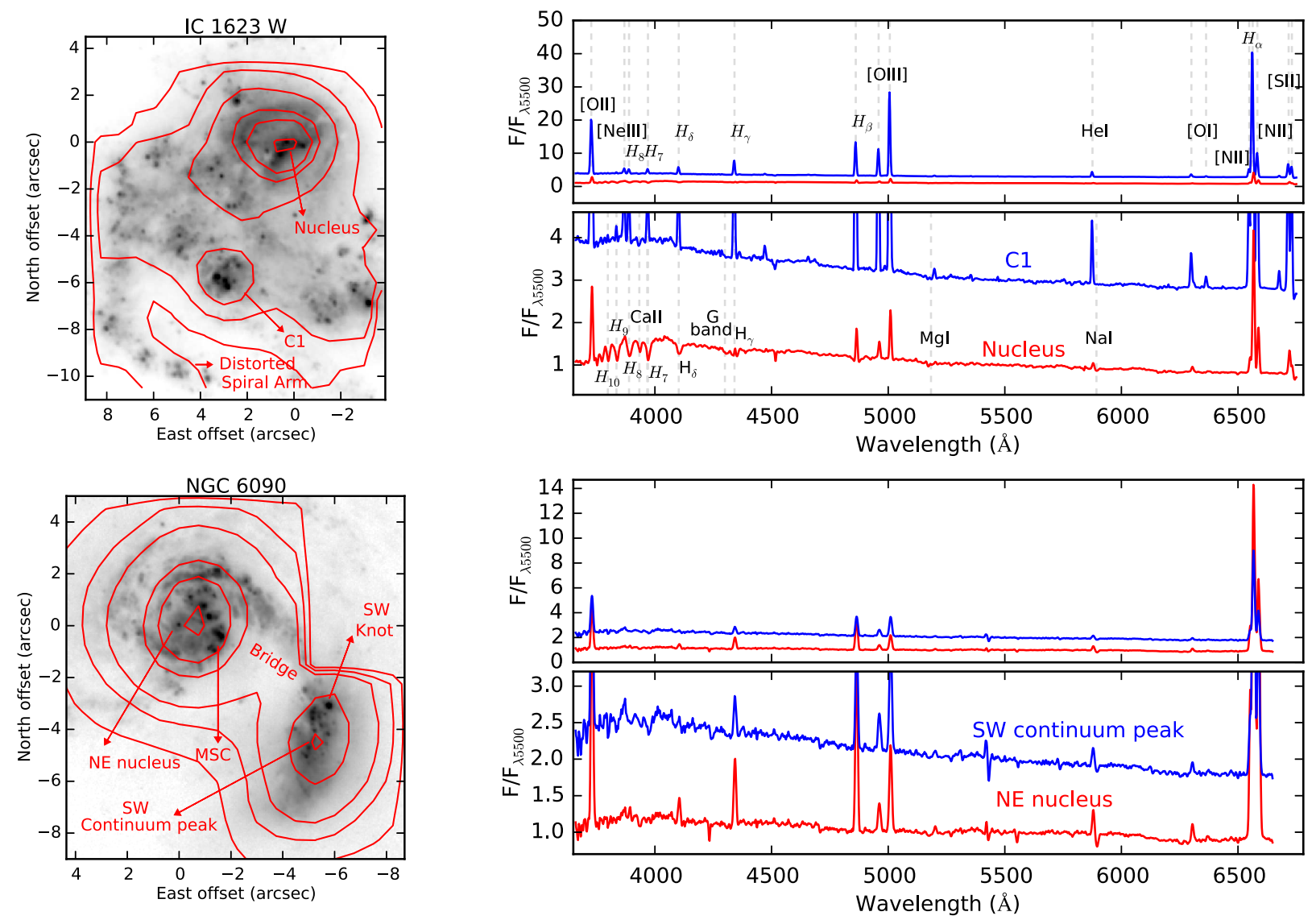

Figure 2. Observations of IC $1623 \mathrm{~W}$ (top panels) and NGC 6090 (bottom panels). Left-hand panel: HST F435W images presented in grey-scale, with the IFS continuum superimposed with red contours. The main regions are labelled. Right-hand panel:spectra of some of these regions, extracted in circular apertures of 2 arcsec radius. The top and bottom panels are the same for each galaxy, but with different $y$-axis ranges.

\subsection{HST imaging data}

In addition to our IFS data, we downloaded from the Hubble Legacy Archive (HLA) ${ }^{1}$ multiwavelength high-resolution images from the $H S T$ in several broad-band filters from FUV to NIR: STIS F25SRF2, STIS F25QTZ, ACS F435W, ACS F814W, NICMOS F110W and NICMOS F160W for IC 1623, and ACS F140LP, ACS F330W, ACS $F 435 W$, ACS $F 814 W$, NICMOS F110W and NICMOS $F 160 W$ for NGC 6090. We used these images to perform aperture photometry on the star clusters present in these early-stage mergers and to roughly determine the stellar population properties. All these images were retrieved in their pipeline reduced state, astrometically corrected, and aligned north up, east left. Their characteristics are summarized in Appendix A, in Tables A1 and A2 for IC 1623 and NGC 6090, respectively.

\subsection{Overview of the data}

In the right-hand panels of Fig. 1, we show the PMAS LArr continuum flux at $5110 \AA$ rest-frame (obtained by averaging between 5050 and $5170 \AA$ ). We have superimposed in contour form the HST $F 435 \mathrm{~W}$ image, smoothed to match the spatial resolution of the IFS $(\sim 1.7$ arcsec). To better identify the main regions, in the left-hand panels of Fig. 2, we show the HST F435W image in grey scale with

\footnotetext{
${ }^{1}$ http://hla.stsci.edu/
}

the IFS continuum superimposed as red contours, along with some spectra extracted in circular apertures of 2 arcsec radius.

In the case of IC $1623 \mathrm{~W}$, the nuclear region, the emission peak $\mathrm{C} 1$ (which corresponds to a giant star-forming region) and the distorted spiral arm are labelled. The $\mathrm{C} 1$ spectrum is dominated by the emission lines and has a blue continuum. We detect broad He II 4686 emission, which is a signature of Wolf-Rayet stars in this system (Crowther 2007). In the nucleus, the line emission is much weaker, and the high-order Balmer lines are visible in absorption, which is a characteristic of intermediate-age (100 Myr to $1 \mathrm{Gyr}$ ) stellar populations.

In the case of NGC 6090, the NGC 6090 NE nucleus is labelled in the bottom left-hand panel of Fig. 2 (and marked with a black dot in the bottom right-hand panel of Fig. 1). To its west, there is the region of multiple star clusters (MSC). In NGC $6090 \mathrm{SW}$, we considered the position of the peak of the IFS continuum to represent the nucleus (marked with a black dot in right-hand panel of Fig. 1). The spectra in the inner 2 arcsec of both nuclei are dominated by line emission, with the SW nucleus having a bluer continuum.

We also note that the brightest point source in the HST images (both optical and NIR) of NGC $6090 \mathrm{SW}$ is the knot located to the north-west of the progenitor, as previously reported by Dinshaw et al. (1999). These authors also found a mismatch between the radio emission peak and this knot, and suggested that it may not be the nucleus of the SW progenitor but a foreground star instead. The IFS has allowed us to confirm that this knot is not a foreground star, because the main absorption lines are at the redshift of the galaxy. 
That is the reason why we cannot discard it as the true nucleus of NGC 6090 SW, and its position will henceforth be shown in the maps with a star. We discuss the minor effect this misidentification would have on our results in Appendix B.

\section{STAR CLUSTER PHOTOMETRY}

Using the HST images from FUV to NIR, we performed aperture photometry of the super star clusters (SSCs) in IC 1623 and NGC 6090. This was to make a rough estimation of their stellar population properties. Clusters were detected with IRAF's DAOFIND task, and photometry performed using the phot task in APPHOT.

According to Alvensleben (2004), a long-wavelength baseline from $U$-band through to the NIR is necessary to determine the properties of star clusters in a reliable way, where the availability of $U$-band photometry is crucial. In fact, we find that the best colour-colour diagrams to break the age-extinction degeneracy are FUV $-F 435 W$ versus $F 435 W-F 814 W$ and $F 435 W-F 814 W$ versus $F 814 W-$ NIC160W. A total of 228 and 156 clusters were detected in IC 1623 and NGC 6090, respectively, with a signal-to-noise ratio SNR $>5$ in FUV, F435W and F814W filters. Analogously, 225 and 117 clusters were detected in IC 1623 and NGC 6090 with an SNR $>5$ in $F 435 W, F 814 W$ and $F 160 W$ filters.

All the clusters with positive detections in at least one of the diagrams are shown in Fig. 3. In the case of IC 1623, the obscured population of clusters in IC $1623 \mathrm{E}$ is shown in red, while that of IC $1623 \mathrm{~W}$ is shown in blue.

The star clusters properties (ages, masses and the amount of dust obscuration) are estimated by comparing their colours and magnitudes with solar metallicity Charlot \& Bruzual (2007, unpublished) simple stellar population (SSP) models. Model colours have been computed using STSDAS.SYNPHOT software.

Details of the methodology, together with the colour-colour, colour-magnitude diagrams are presented in the Appendix A. Here we summarize the main results.

IC 1623: Most IC $1623 \mathrm{~W}$ clusters are very young ( $<200 \mathrm{Myr})$ and affected by low to almost no foreground extinction $(<1 \mathrm{mag})$. In contrast, the clusters in IC $1623 \mathrm{E}$ are affected by 2-6 mag of extinction. For the only IC 1623 E cluster emitting in FUV, we derive an age below $10 \mathrm{Myr}$, and $A_{V}=2-3 \mathrm{mag}$. This suggests that, although affected by large amounts of dust extinction, some clusters in IC $1623 \mathrm{E}$ are as young as or younger than those in IC $1623 \mathrm{~W}$. Moreover, the clusters in IC $1623 \mathrm{~W}$ have masses between $10^{5}$ and $10^{7} \mathrm{M}_{\odot}$, while those in IC $1623 \mathrm{E}$ are more massive $\sim 10^{7}$ $10^{8} \mathrm{M}_{\odot} \cdot{ }^{2}$ The two clusters with masses as high as $10^{9} \mathrm{M}_{\odot}$ are spatially coincident with the two nuclei in IC $1623 \mathrm{E}$ reported by (Knop et al. 1994). A rough estimate of the total mass in clusters in IC $1623 \mathrm{~W}$ is $\sim 9 \times 10^{8} \mathrm{M}_{\odot}$.

NGC 6090: Most of the clusters in NGC 6090 are also young ( $<300 \mathrm{Myr}$ ) and affected by $<1$ mag extinction, but some of them could have $A_{V}$ up to 2 mag. In general, NGC 6090 clusters have masses between $10^{5}$ and $10^{7} \mathrm{M}_{\odot}$. We find that the two 'clusters' with higher masses (around $10^{7}-10^{8} \mathrm{M}_{\odot}$ ) are NGC $6090 \mathrm{NE}$ nucleus and NGC $6090 \mathrm{SW}$ nucleus. The total stellar mass in clusters in NGC 6090 is $\sim 2 \times 10^{9} \mathrm{M}_{\odot}$.

As noted in Section A5, star cluster photometry allow us to trace the star formation that occurred in the last $<500 \mathrm{Myr}-1 \mathrm{Gyr}$ (at best, for no extinction), but not in previous epochs. Although spectral

\footnotetext{
${ }^{2}$ Probably knots with masses higher than $10^{7} \mathrm{M}_{\odot}$ are clumps of several clusters rather than individual unique clusters.
}

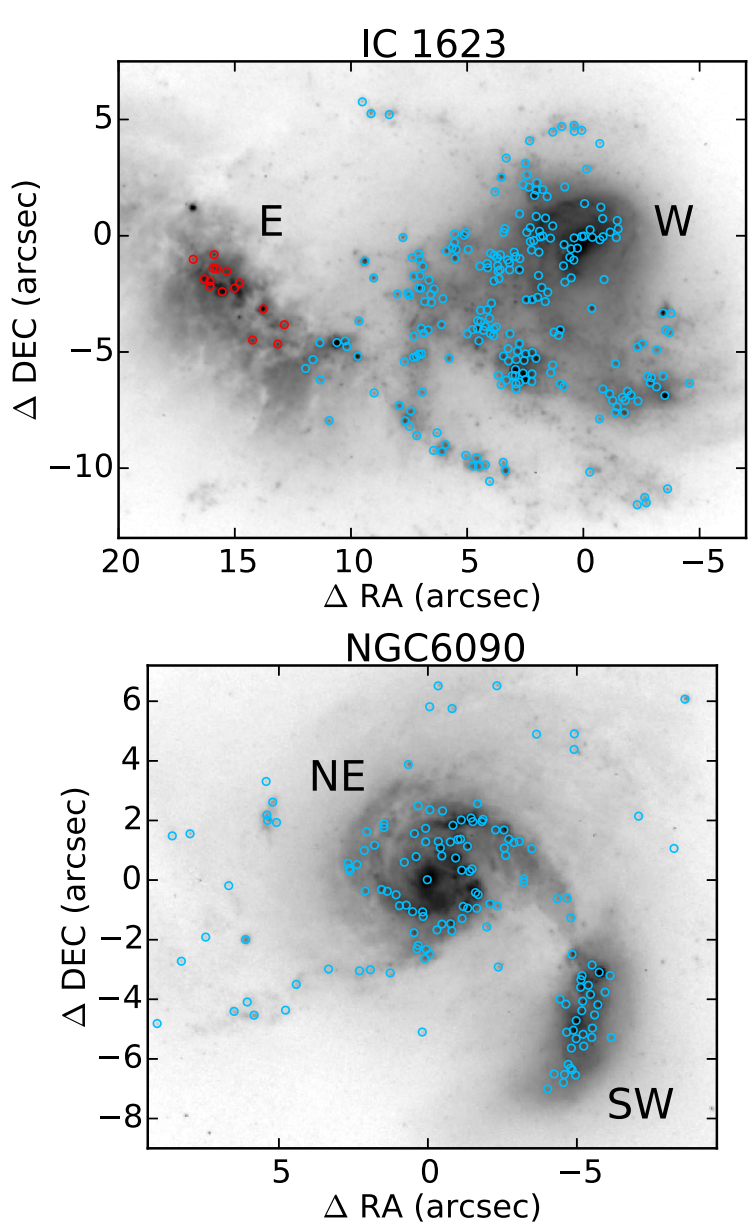

Figure 3. F814W images of IC 1623 (top panel) and NGC 6090 (bottom panel). North is up, east to left. The detected clusters are marked with blue circles. In the case of IC 1623, we used red colour for the clusters in IC 1623 $\mathrm{E}$, and blue for the ones in IC $1623 \mathrm{~W}$, because they clearly represent two different populations in the colour-colour and colour-magnitude diagrams.

synthesis reveals that intermediate-age stellar populations (ISP) are not important contributors in these galaxies (Fig. 8, Section 4.7), the star cluster detection limits are relevant to be known when studying more advanced merger stages, where a significant intermediate-age population could be present.

\section{STELLAR POPULATIONS}

In this section, we use the stellar continuum shape to characterize the spatially resolved stellar population properties and constrain the star formation histories (SFHs) of IC 1623 and NGC 6090.

In order to quantify the effects of mergers on the properties of the stellar populations, the results of our LIRGs are compared to control samples of CALIFA spirals from González Delgado et al. (2015a), which have been calibrated with version 1.5 of the reduction pipeline, explained in detail in the Data Release 2 article (García-Benito et al. 2015). The comparisons are made with $\mathrm{Sbc/Sc}$ spirals because the progenitor galaxies of LIRGs are gasrich spirals without significant bulges (Sanders \& Mirabel 1996; Kim et al. 2013), and because they have stellar masses similar to IC 1623 and NGC 6090. We have defined two possible control samples. On one hand, the average of all the Sbc galaxies in 
CALIFA, because they approximately cover the same mass range as our LIRGs, with $\left\langle\log M_{\star}^{\text {Sbc }}\left(\mathrm{M}_{\odot}\right)\right\rangle=10.70 \pm 0.34$. On the other hand, using later Sc galaxies, but averaging only those in a mass range compatible with our LIRGs, $\left\langle\log M_{\star}^{\mathrm{Sc}}\left(\mathrm{M}_{\odot}\right)\right\rangle=10.45-10.85$. There are $14 \mathrm{Sc}$ galaxies in the CALIFA sample meeting this requirement, and $\sim 70$ Sbc galaxies.

\subsection{Methodology}

Our method to extract the stellar population information from the IFS data cubes is based on the full spectral synthesis approach. This technique has been extensively tested and applied (Cid Fernandes \& González Delgado 2010; Cid Fernandes et al. 2013, 2014; González Delgado \& Cid Fernandes 2010; Pérez et al. 2013; González Delgado et al. 2014), and it has been proven to reduce the agemetallicity degeneracy (Sánchez-Blázquez et al. 2011). It is also notable that despite the diversity of spectral synthesis methods, substantial changes in the results are more likely to come from revisions in the input data and from updates in the base models, which are the single most important ingredient in any spectral synthesis analysis (Cid Fernandes et al. 2013). The general consensus in the field is that uncertainties in the results for individual objects average out for large statistical samples (Panter et al. 2007). With IFS, each galaxy is a statistical sample per se, and even if the results for single spaxels (or zones) are uncertain, the overall trends should be robust.

We have analysed the stellar population properties with the STARLIGHT code (Cid Fernandes et al. 2005), which fits an observed spectrum $\left(\mathrm{O}_{\lambda}\right)$ in terms of a model $\left(\mathrm{M}_{\lambda}\right)$ built by a nonparametric linear combination of $N_{\star}$ SSPs from a base spanning different ages $(t)$ and metallicities $(Z)$. Dust effects are modelled as a foreground screen with a Calzetti et al. (2000) reddening law with $R_{V}=4.5$, assuming the same reddening for all the SSP components. Kinematical effects are also accounted for by assuming a Gaussian line-of-sight velocity distribution. The fits were carried out in the rest-frame $3650-6950 \AA$ interval. Windows around the [O I $],\left[\mathrm{O}_{I I}\right],\left[\mathrm{O}_{\mathrm{III}}\right],\left[\mathrm{N}_{\mathrm{II}}\right], \mathrm{He}_{\mathrm{I}}$ and Balmer series from $\mathrm{H} \alpha$ to $\mathrm{H} \epsilon$ emission lines were masked in all fits. Because of its interstellar absorption component, the Na I D doublet was also masked. The process is similar to that previously applied in CALIFA data (Cid Fernandes et al. 2013, 2014).

The results reported in this paper rely on the GM base (Cid Fernandes et al. 2013, hereafter CF2013) of SSP spectra, which combines the GRANADA models of González Delgado et al. (2005) for $t<63$ Myr with those of Vazdekis et al. (2010). They are based on the Salpeter initial mass function (IMF). It contains 156 SSPs, comprising four metallicities, $Z=0.2,0.4,1$ and $1.6 \mathrm{Z}_{\odot}$, and 39 ages between $t=10^{6}$ and $1.4 \times 10^{10} \mathrm{yr}$. For comparison purposes, we have also performed the spectral synthesis using the CB model base, which is built from an update of the Bruzual \& Charlot (2003) models, replacing STELIB by a combination of the MILES (Sánchez-Blázquez et al. 2006) and GRANADA (Martins et al. 2005) spectral libraries (the same ones used in base GM). The IMF is that of Chabrier (2003) and it comprises 160 SSPs of four metallicities, $Z=0.2,0.4,1$ and $2.5 \mathrm{Z}_{\odot}$, and 40 ages between $t=10^{6}$ and $1.4 \times 10^{10} \mathrm{yr}$.

We discarded from the analysis all the spaxels with $\mathrm{SNR}<5$ in the continuum, measured in the rest-framed window between 5590 and $5680 \AA$. Also, the index

$\bar{\Delta}=\frac{1}{N_{\lambda}^{\mathrm{eff}}} \sum_{\lambda} \frac{\left|\mathrm{O}_{\lambda}-\mathrm{M}_{\lambda}\right|}{\mathrm{M}_{\lambda}}$ proposed by CF2013 has been used to quantify the quality of the spectral fits (see their section 5.1). Again, $\mathrm{O}_{\lambda}$ and $\mathrm{M}_{\lambda}$ are the observed and model spectra, respectively, and the sum is carried over the $N_{\lambda}^{\text {eff }}$ wavelengths actually used in the fit, i.e. discarding masked, flagged and clipped pixels. $\bar{\Delta}=10$ per cent is a reasonable qualitycontrol limit. Typical median values of $\sim 3$ per cent and $\sim 5$ per cent are found in IC $1623 \mathrm{~W}$ and NGC 6090, respectively. From now on, only the spaxels with $\bar{\Delta}$ below the control limit are shown in the maps, and considered in the analysis.

\subsection{Stellar mass}

An important STARLIGHT output is the stellar mass, which is calculated taking into account the spatial variations of the mass-to-light ratio. The total stellar mass obtained from the sum of all spaxels is $M=3.9 \times 10^{10}$ and $6.8 \times 10^{10} \mathrm{M}_{\odot}$ for IC $1623 \mathrm{~W}$ and NGC 6090 , respectively (for Salpeter IMF). This is the mass locked in stars today. Also counting the mass returned by stars to the ISM $M^{\prime}=5.5 \times 10^{10}$ and $9.6 \times 10^{10} \mathrm{M}_{\odot}$ were involved in star formation for IC $1623 \mathrm{~W}$ and NGC 6090, respectively.

Since, in LIRGs, the optical light is dominated by young stellar populations (YSP) and the extinction may be important in some regions, we could be underestimating the mass of the old stars. The OSP emit the bulk of their light in the 1-3 $\mu \mathrm{m}$ NIR window, and assuming that they are the principal contributors to the IR light, we have determined an alternative estimate of the stellar mass using MIR data from Spitzer at 3.6 and $4.5 \mu \mathrm{m}$, following the approach proposed by Jarrett et al. (2013). The global mass-to-light ratio at $3.6 \mu \mathrm{m}\left(\zeta_{3.6}^{d}\right)$ can be expressed as

$\log \zeta_{3.6}^{d}\left(\mathrm{M}_{\odot} / \mathrm{L}_{\odot}\right)=-0.22+3.42([3.6]-[4.5])$,

where [3.6] and [4.5] are the magnitudes in the 3.6 and $4.5 \mu \mathrm{m}$ bands. This expression was calculated under several assumptions that we have to take into account. On one hand, it considers the SSPs of Bruzual \& Charlot (2003) with a Chabrier IMF. On the other hand, it considers that the average mass-to-light ratio (at $3.6 \mu \mathrm{m}$ ) for strictly old stars is $\log \zeta_{3.6}=-0.23 \pm 0.1$ dex, as determined by Meidt et al. (2012, 2014), and valid in the range of metallicities covered by our models. With this procedure, we found that the stellar mass derived from the MIR is $2.8 \times 10^{10}$ and $4.2 \times 10^{10} \mathrm{M}_{\odot}$ for IC $1623 \mathrm{~W}$ and NGC 6090, respectively (Chabrier IMF), in good agreement with our stellar masses derived from the spectral synthesis, if MIR masses are scaled by a factor of $\sim 1.8$ as expected due to the change in the IMF.

This agreement between the mass estimations derived from optical spectra and MIR imaging confirms that for IC $1623 \mathrm{~W}$ and NGC 6090, we are not underestimating the stellar masses with spectral synthesis, and also indicates that the extinction estimates are approximately correct.

\subsection{Stellar mass surface density}

In Fig. 4, we show the stellar mass surface density $\left(\mu_{\star}\right)$ maps for IC $1623 \mathrm{~W}$ (upper left-hand panel) and NGC 6090 (upper right-hand). The black dashed circles indicate the position of 1 half-light radius $(\mathrm{HLR})^{3}$ in IC $1623 \mathrm{~W}$ and NGC $6090 \mathrm{NE}$, and 0.5 HLR in NGC

\footnotetext{
${ }^{3}$ The HLR is defined as the axis length of the circular aperture, which contains half of the total light of the galaxy at the rest-frame wavelength. It was obtained from the continuum flux image at $5635 \AA$ using the PYCASso package.
} 

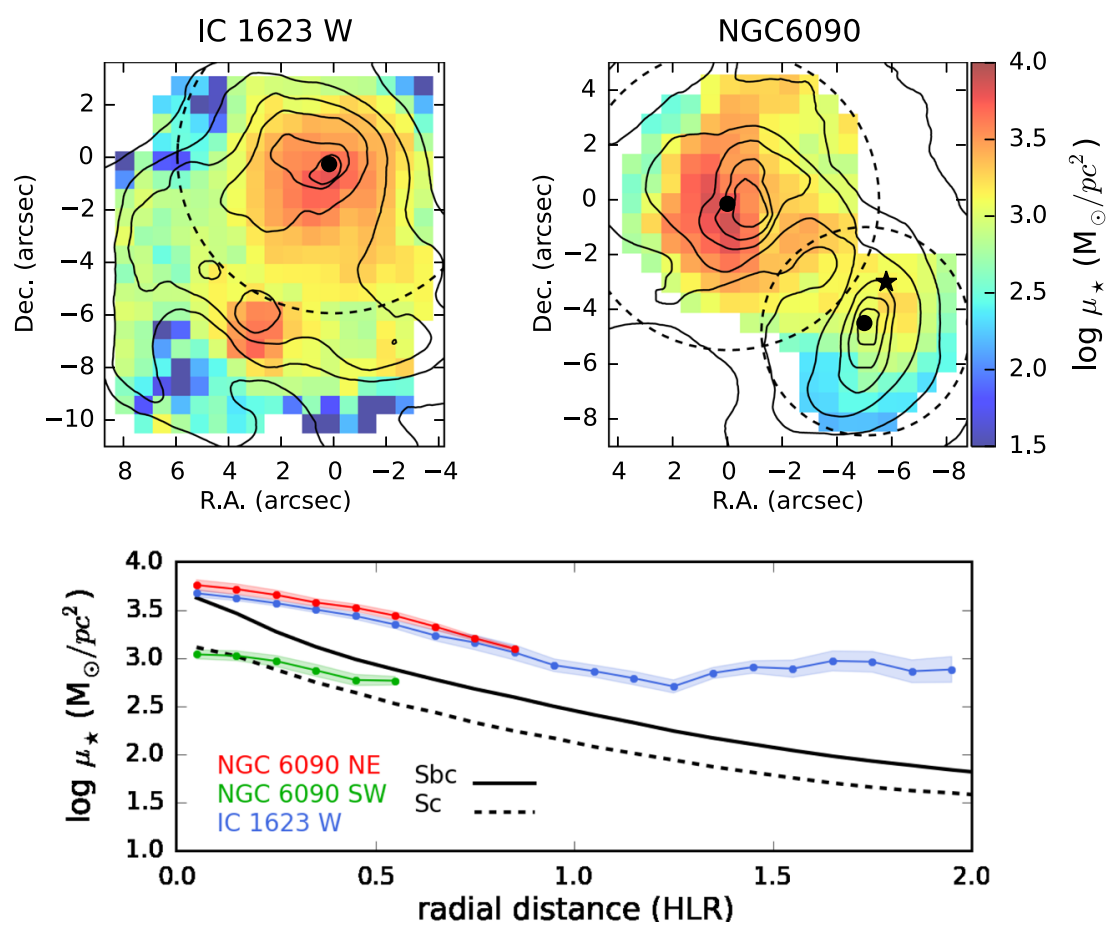

Figure 4. Observed stellar mass surface density for IC 1623 (upper left-hand panel) and NGC 6090 (upper right-hand panel). The colour scale is the same for both maps. The black dashed lines indicate the position of 1 half-light radius (HLR) in IC $1623 \mathrm{~W}$ and NGC 6090 NE, and 0.5 HLR in NGC 6090 SW. The solid contours correspond to the smoothed HST F435W images. Lower panel: mean radial profiles of the stellar mass surface density as a function of the radial distance in HLR, in red for NGC $6090 \mathrm{NE}$, green for NGC $6090 \mathrm{SW}$ and blue for IC $1623 \mathrm{~W}$. The uncertainties, calculated as the standard error of the mean, are shaded in light red, green and blue, respectively. For comparison, the black lines are the average profiles for Sbc (solid) and Sc (dashed) spiral galaxies in CALIFA (GD2015), with masses consistent with our LIRGs.

6090 SW. 1 HLR 2.5, 3.4 and $5.0 \mathrm{kpc}$ for IC $1623 \mathrm{~W}$, NGC 6090 NE and NGC $6090 \mathrm{SW}$, respectively, on average, while for the Sbc and Sc control galaxies, $1 \mathrm{HLR} \sim 5.0$ and $4.1 \mathrm{kpc}$, respectively.

The 2D maps of the stellar population properties were azimuthally averaged to study their radial variations using PYCASSO (CF2013). Radial apertures 0.1 HLR in width were used to extract the profiles. Expressing radial distances in units of HLR allows the profiles of the early-stage mergers to be compared on a common metric with the control samples of Sbc and Sc galaxies. The $\mu_{\star}$ profiles are shown in the lower panel: in red for NGC $6090 \mathrm{NE}$, NGC $6090 \mathrm{SW}$ in green and IC $1623 \mathrm{~W}$ in blue. The uncertainties are shaded in red, green and blue, respectively. They represent the standard error of the mean, calculated as the standard deviation divided by the square root of the number of points in each distance bin, with 0.2 HLR of width. As it is normalized by the square root of the number of points/spaxels in each HLR bin, the differences in the uncertainties between the inner and outer regions are visually not so strong, as the larger the radius, the larger is $N$. In terms of dispersion (=standard deviation), we find that with respect to IC $1623 \mathrm{~W}$ centre $(N=8)$, the dispersion is a factor of 2.3 larger at 0.5 $\operatorname{HLR}(N=41), 2.4$ larger at $1 \mathrm{HLR}(N=47)$ and 1.5 larger at 2 HLR $(N=18)$. In the case of NGC $6090 \mathrm{NE}$, the dispersion is a factor of 2.3 larger at $0.5 \operatorname{HLR}(N=42)$ than at the centre $(N=8)$, and for NGC $6090 \mathrm{SW}$ is a factor 2.5 larger at $0.5 \operatorname{HLR}(N=74)$ than at the centre $(N=12)$. The black lines are the profiles from Sbc (solid) and Sc (dashed) spiral galaxies from CALIFA (González Delgado et al. 2015a, hereafter GD2015).

The highest values of the mass surface density are reached in IC $1623 \mathrm{~W}$ nucleus and in the cluster concentration $\mathrm{C} 1$, with a logarithmic value of around 3.7 for $\mu_{\star}$ in units of $\mathrm{M}_{\odot} \mathrm{pc}^{-2}$. From the radial profile, we find a negative gradient with distance, going from $\log \mu_{\star}=3.7$ at the nucleus to 2.8 at $1.2 \mathrm{HLR}$, then increasing again up to $\log \mu_{\star}=3.0$ at 2 HLR.

In NGC 6090, the stellar mass surface density in the NE progenitor is significantly higher than in the SW. The region with the highest value, $\log \mu_{\star} \sim 3.8$, is located to the south-east of the NGC $6090 \mathrm{NE}$ nucleus. In NGC $6090 \mathrm{SW}, \log \mu_{\star}$ is higher towards the north. From the radial profile, we also found a negative gradient of the stellar mass density with distance: $\log \mu_{\star}$ goes from 3.8 to 3.1 at 0.9 HLR for NGC $6090 \mathrm{NE}$, and from 3.0 to 2.8 at $0.6 \mathrm{HLR}$, for NGC 6090 SW.

The central density in IC $1623 \mathrm{~W}$ and NGC $6090 \mathrm{NE}$ is similar to the central density of Sbc galaxies, while NGC $6090 \mathrm{SW}$ is more similar to Sc. We have compared the inner gradients of the stellar mass surface density (from 0 to $1 \mathrm{HLR}$, as defined in equation 6 of GD2015) in early-stage mergers and in the control spirals. We find $\Delta_{\text {in }} \log \mu_{\star}=-0.66,-0.79,-0.99$ and -1.15 for NGC $6090 \mathrm{NE}$ (in $0.9 \mathrm{HLR}$ ), IC $1623 \mathrm{~W}, \mathrm{Sc}$ and Sbc, respectively. In the case of IC $1623 \mathrm{~W}$, we can also compare the outer gradient (from 1 to 2 HLR, defined as in equation 7 of GD2015), $\Delta_{\text {out }} \log \mu_{\star}=0,-0.54$ and -0.64 for IC $1623 \mathrm{~W}, \mathrm{Sc}$ and Sbc galaxies, respectively. We find that the early-stage merger radial profiles of $\mu_{\star}$ are flatter than in spirals, but not so significantly if we consider the dispersion of the control samples, between 0.24 and 0.28 dex (GD2015). Moreover, we note that given the recent star formation in IC $1623 \mathrm{~W}$, its HLR is $\sim$ half the HLR of the control samples, and the apparent flattening of the mass density profile may be also due to this, instead of representing a real evolutionary change, at least in the inner 1 HLR. This does not affect NGC $6090 \mathrm{NE}$ and SW, as their HLRs are similar to Sbc/Sc galaxies. 

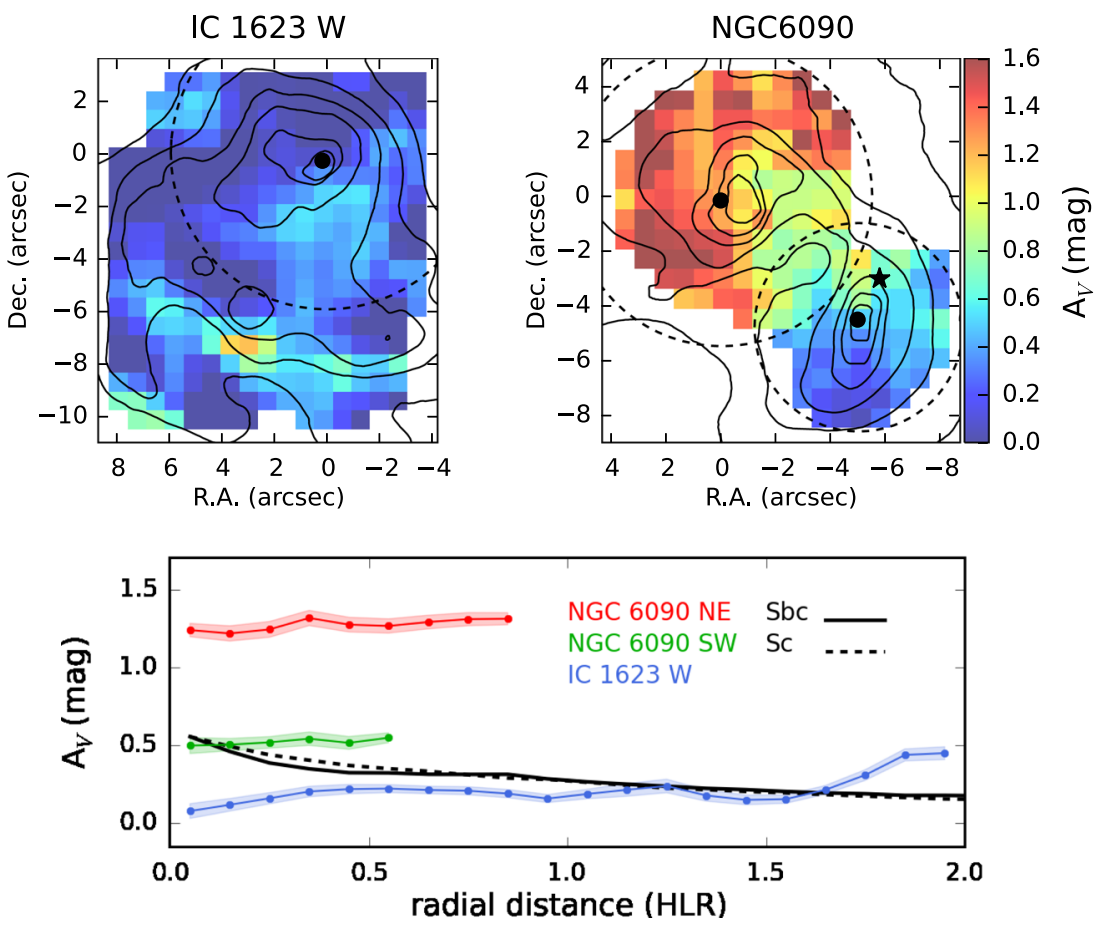

Figure 5. As Fig. 4 but for stellar dust extinction.

\subsection{Stellar dust extinction}

STARLIGHT-based $A_{V}$ maps are shown in Fig. 5. The scale is the same for both systems to facilitate the comparison.

IC $1623 \mathrm{~W}$ (upper left-hand panel) has little extinction, with $A_{V}$ below $0.4 \mathrm{mag}$ nearly everywhere. From the radial profile, we found a slight but non-uniform increase of the average extinction from $0.06 \mathrm{mag}$ in the nucleus to $0.2 \mathrm{mag}$ at $1.5 \mathrm{HLR}$. The regions most affected by extinction are located near areas where $\mathrm{H}$ II regions accumulate, such as $\mathrm{C} 1$, where the extinction reaches values up to $1.2 \mathrm{mag}$. As an explanation, we suggest that the enhancement of $A_{V}$ could be due to the accumulation of dust swept by the winds and supernovae of the star-forming regions, at least in some locations. Another possibility is that the interaction itself has led to the accumulation of dust in these regions.

In NGC $6090 \mathrm{NE}$ (upper right-hand panel), $A_{V}$ ranges from 1.2 to $1.6 \mathrm{mag}$, while in NGC $6090 \mathrm{SW}$, it runs from $0.6 \mathrm{mag}$ in the north to about $0.2 \mathrm{mag}$ in the south. To the west of NGC $6090 \mathrm{NE}$ and in the bridge between the two galaxies, the dust extinction has an intermediate value of $0.8-0.9 \mathrm{mag}$. There is $\sim 1 \mathrm{mag}$ difference in the stellar dust extinction between the two progenitors. When averaging (lower panel), we do not find significant gradients of the dust distribution across the main bodies of the individual galaxies, reflected in the nearly flat radial profiles. We note that in these two early-stage merger systems, one of the progenitors is significantly more obscured than the other. In these cases, it happens that the eastern progenitor is more obscured, although the difference is higher for IC 1623 than for NGC 6090.

The radial profiles of $A_{V}$ in IC 1623 and NGC 6090 are flat. This is why the $\mu_{\star}$ profiles shown in the previous section resemble the surface brightness profiles (not shown). In contrast, typical spiral galaxies show a negative radial gradient independent of the morphology (GD2015).

\subsection{Ages}

The simplest way to quantify the SFH of a system is to compress the age distribution encoded in the light population vectors to their first moments. For this purpose, we will use the following definition for the mean stellar age:

$\langle\log a g e\rangle_{\mathrm{L}}=\sum_{t, Z} x_{t, Z} \log t$

where $x_{t, Z}$ is the fraction of light at the normalization wavelength (5635 $\AA$ ) due to the base population with age $t$ and metallicity $Z$.

The mean stellar age maps are shown in Fig. 6. The scale is the same for both maps to facilitate the comparison and goes from $\langle\log \text { age }\rangle_{\mathrm{L}}=6.0-9.0$.

In IC $1623 \mathrm{~W}$, the nuclear regions are clearly older than the outer parts. From the radial profile, we find a negative trend of the mean age with distance: from $\sim 300 \mathrm{Myr}$ in the nucleus to $\sim 30 \mathrm{Myr}, 1.2$ HLR away. From 1.2 to 2 HLR, the age remains approximately constant at 15-40 Myr, indicating that the IC $1623 \mathrm{~W}$ disc is still forming stars.

In NGC 6090, the older spaxels are located in the outskirts for both progenitors, but especially for the SW galaxy, where they reach ages up to $\sim 300 \mathrm{Myr}$. The youngest spaxels ( $\sim 50 \mathrm{Myr}$ ) are located in the bridge. When averaging, we found that both progenitors have the same mean ages, $\langle\log \text { age }\rangle_{\mathrm{L}} \sim 50-100 \mathrm{Myr}$.

In both early-stage merger systems, the age profiles are significantly flatter than in $\mathrm{Sb}$ to Sc galaxies (GD2015). Even for IC $1623 \mathrm{~W}$, where we find a negative gradient of $-280 \mathrm{Myr}$ in the inner $1 \mathrm{HLR}$, it is still much lower than the $-2.4 \mathrm{Gyr}(-620 \mathrm{Myr})$ gradient in the inner $1 \mathrm{HLR}$ of $\mathrm{Sbc}(\mathrm{Sc})$ galaxies. 

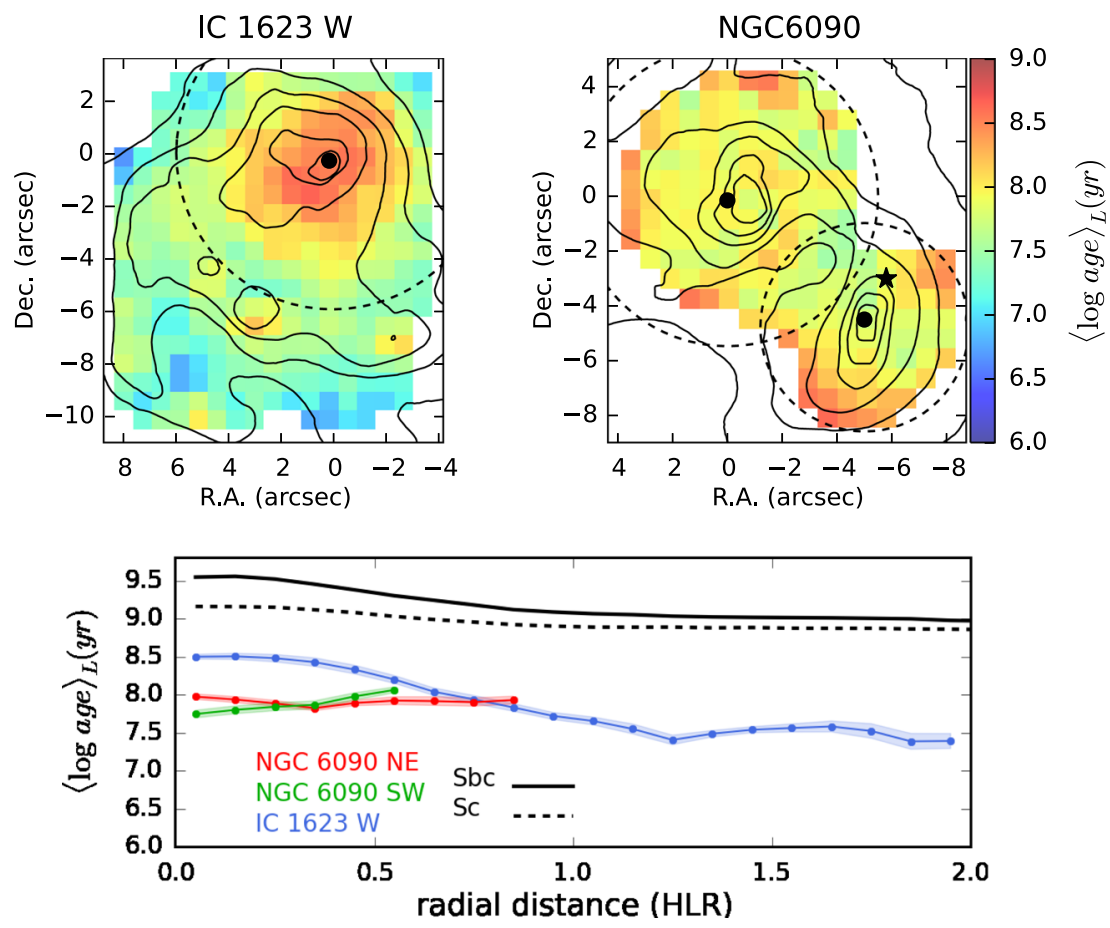

Figure 6. As Fig. 4 but for mean light-weighted stellar ages.

\subsection{Metallicity}

The mean metallicity has been obtained in a similar way to the mean light-weighted ages, but weighing by the mass:

$\left\langle\log Z_{\star}\right\rangle_{\mathrm{M}}=\sum_{t, Z} \mu_{t, Z} \log Z$,

where $\mu_{t, Z}$ is the mass fraction in the base population with age $t$ and metallicity $Z$. This is the same definition adopted in GD2015. The mass-weighted metallicity maps are shown in Fig. 7. The scale range in both maps is the same, and spans the full range of the base (from -0.7 to +0.2 in log-solar units).

As can be seen in Fig. 7, the scatter is large, maybe due to the weakness of the metallic absorption lines in the spectra. Moreover, our results could be affected by the age-metallicity degeneracy, although the spectral synthesis has been proven to be better than the index method to break it (Sánchez-Blázquez et al. 2011).

Focusing on the radial averages, it seems that IC $1623 \mathrm{~W}$, NGC $6090 \mathrm{NE}$ and SW have global mean metallicities around $\sim 0.6 \mathrm{Z}_{\odot}$ (but ranging from 0.4 to $0.8 \mathrm{Z}_{\odot}$ ). We find that beyond $0.7 \mathrm{HLR}$ in IC $1623 \mathrm{~W}$, the metallicities are similar to those of Sbc galaxies, but in the central regions (in the inner 0.5 HLR), the metallicities are smaller, more similar to Sc galaxies. Something similar happens in NGC $6090 \mathrm{NE}$, where a decrease in the metallicity below 0.4 HLR is found. The metallicity radial profiles in the inner 1 HLR of these three galaxies have flat or even slightly positive gradients, contrary to what happens in spirals earlier than Sc, which show a negative gradient (GD2015). However, we cannot claim a dilution or interpret this further, given the large dispersion of up to $0.25 \mathrm{dex}$ in the metallicity of the control samples.

\subsection{Contribution of young, intermediate and old populations}

Population synthesis studies in the past found that a useful way to summarize the SFH is to condense the age distribution encoded in the population vector into age ranges. This strategy originated in methods based on fitting equivalent spectral indices (Bica, Arimoto \& Alloin 1988; Cid Fernandes, Leão \& Lacerda 2003), but it was also applied to full spectral fits (González Delgado et al. 2004). We follow this strategy by dividing into: YSP $(t \leq 0.14 \mathrm{Gyr})$, ISP $(0.14<t \leq 1.4 \mathrm{Gyr})$ and OSP $(t>1.4 \mathrm{Gyr})$. We select these three age bins to correspond to the lifetimes of populations with distinctly different optical line and continuum features.

Fig. 8 presents the maps (left-hand panel) and radial profiles (right-hand panel) of the light fractions due to YSP, ISP and OSP $\left(x_{\mathrm{Y}}, x_{\mathrm{I}}\right.$ and $\left.x_{\mathrm{O}}\right)$. IC $1623 \mathrm{~W}$ is shown in the top panels, and NGC 6090 in the bottom panels. We find that both early-stage merger systems are dominated by YSP over their full observed extents.

In IC $1623 \mathrm{~W}$, we find that the contribution of the YSP to light is around 90 percent everywhere, except in the centre, where it is around 50 per cent. In contrast, in Sbc galaxies, the YSP contribution is much lower: $\sim 10$ per cent in the centre and 20 per cent in the disc. In IC $1623 \mathrm{~W}$, the ISP has a significant contribution only in the inner $0.3 \mathrm{HLR}$, with contributions of 30-40 per cent, higher but still similar to Sbc galaxies, $\sim 20$ per cent. The contribution of OSP is everywhere below 20 per cent in IC $1623 \mathrm{~W}$, while in Sbc galaxies, it ranges from 70 per cent in the nucleus to 40 per cent at 2 HLR.

In NGC 6090, we find that almost all spaxels have YSP contribution above 70 per cent (much higher than the 10-20 per cent in Sbc galaxies), except for some spaxels located at the easternmost end of NGC $6090 \mathrm{NE}$ and at the south edge of NGC $6090 \mathrm{SW}$, where the contribution of ISP is also significant. However, the average contribution of ISP is small, below 10 per cent for both progenitors, smaller than the 20-40 per cent typical of Sbc galaxies. The OSP contributes around 20 per cent of the light of both progenitors over their full extents, much smaller than its contribution in Sbc galaxies.

Analogously, in Fig. 9, we present the maps (left-hand panel) and radial profiles (right-hand panel) of the mass contribution by percentage of the YSP, ISP and OSP $\left(m_{\mathrm{Y}}, m_{\mathrm{I}}\right.$ and $\left.m_{\mathrm{O}}\right)$. For both IC 

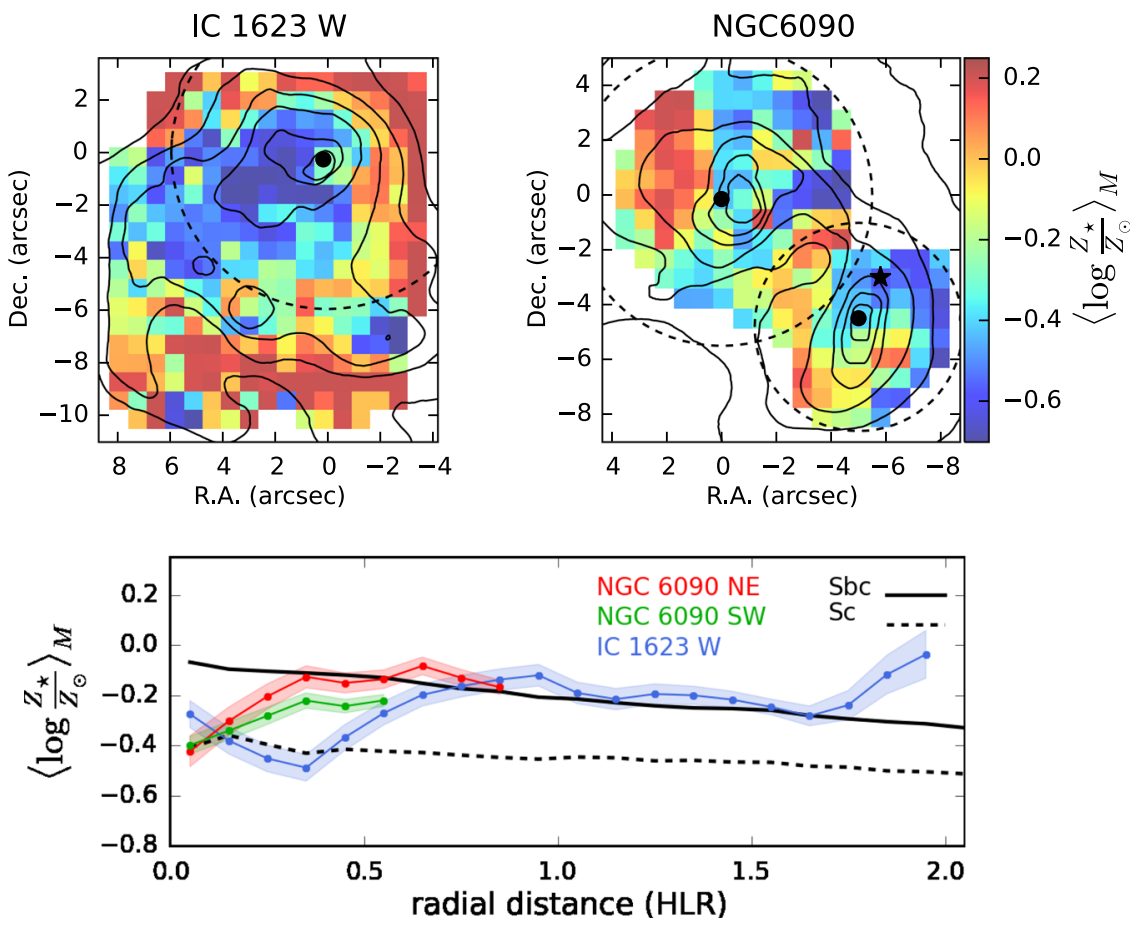

Figure 7. As Fig. 4 but for mean mass-weighted metallicity in solar units.
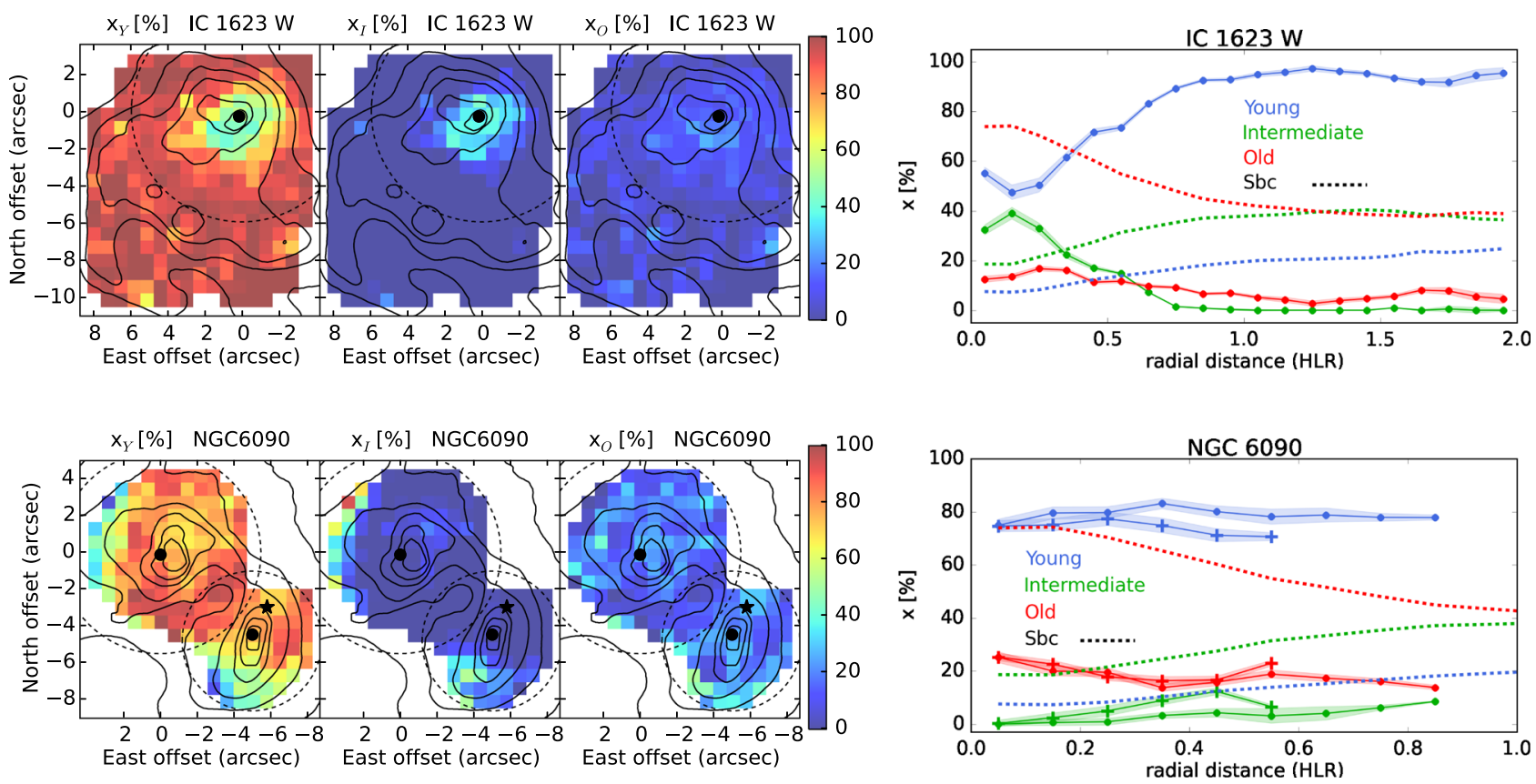

Figure 8. Left-hand panels: contribution in percentage of (a) young $t<140 \mathrm{Myr}$, (b) intermediate (140 Myr $<t<1.4 \mathrm{Gyr}$ ) and (c) old $t>1.4 \mathrm{Gyr}$ stellar populations to the observed light at $5635 \AA$. Right-hand panels: radial profiles of the light contributions from (a) young $x_{\mathrm{Y}}$ (blue), (b) intermediate $x_{\mathrm{I}}$ (green) and (c) old populations $x_{\mathrm{O}}$ (red), with the radial distance in HLR. For comparison, the dotted lines with the same colours are the average profiles for the Sbc galaxies in CALIFA. Top panels: IC 1623 W. Bottom panels: NGC 6090. The NGC 6090 NE radial profile has circle symbols, while the NGC 6090 SW profile has plus symbols. We find that both early-stage merger systems are clearly dominated by YSP across the full observed extents of the systems.

$1623 \mathrm{~W}$ (top panels) and NGC 6090 (bottom panels), most of the mass is in OSP.

In IC $1623 \mathrm{~W}$, this is true everywhere, except for some spaxels in the outer regions, which are completely dominated by young components. The radial profiles of the OSP and YSP mass contributions evolve inversely to each other. In a non-uniform way, the OSP contribution decreases from 80 per cent in the nucleus to 40 per cent at 2 HLR. Conversely, the mass in YSP grows from 5 percent in the nucleus to 60 per cent at 2 HLR. In terms of the mass in ISP, they range from 15 per cent in the nucleus to almost 0 beyond 0.7 HLR. 

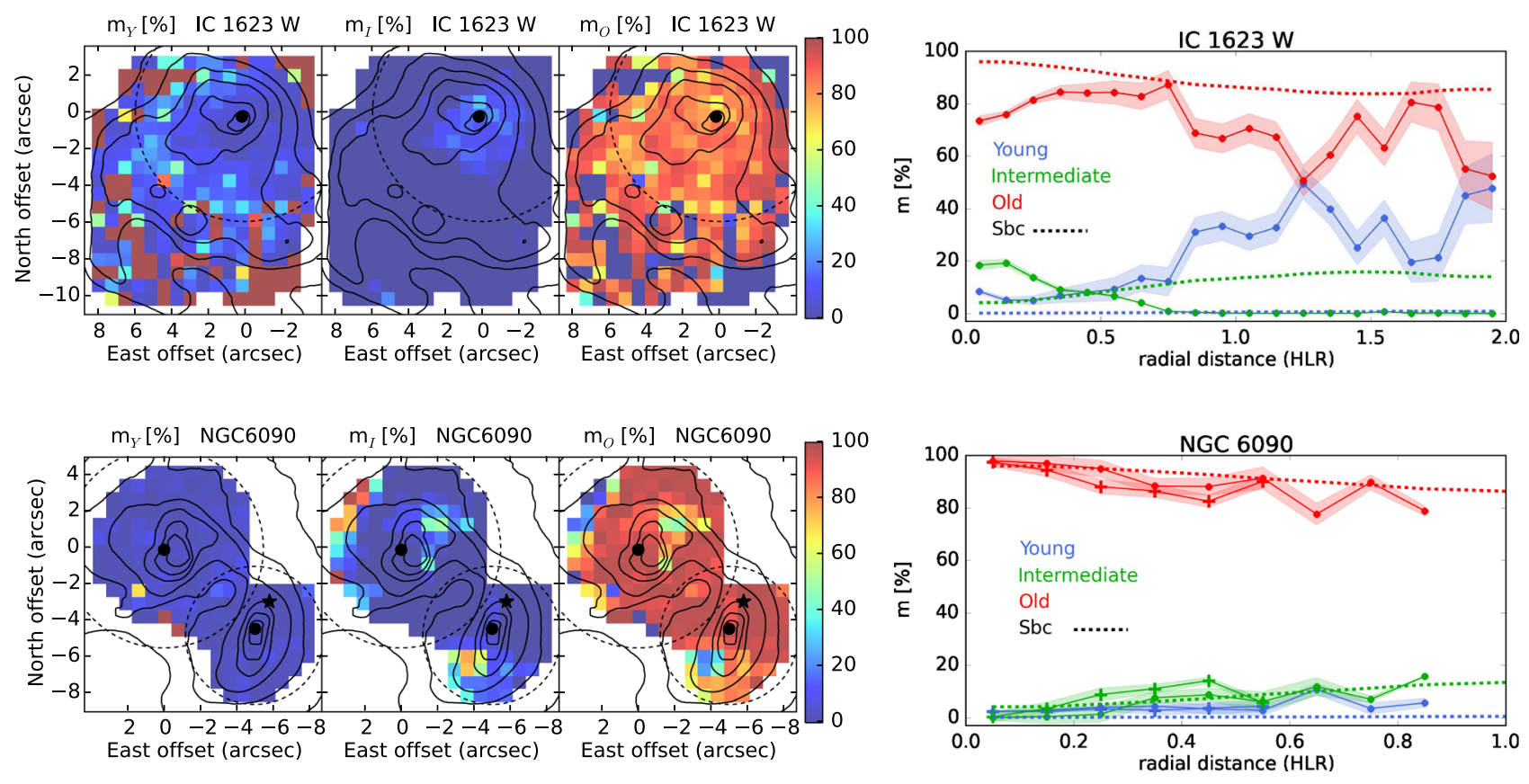

Figure 9. As Fig. 8 but for contributions to the stellar mass.

The same happens with NGC 6090, with the OSP dominating the mass everywhere except in the easternmost end of NGC $6090 \mathrm{NE}$, in some spaxels to the north-west of NGC 6090 NE nucleus, and to the south of NGC $6090 \mathrm{SW}$, where the contributions of ISPs are also large (50-80 per cent, and up to 90 per cent in some spaxels). From the radial profile, we see that for both progenitors, the OSP is responsible for more than 80 per cent of the mass, and this is true over the full observed extent of the system. The fractions of stellar mass in YSP and ISP are comparatively low.

We have also computed the total masses in YSP, ISP and OSP. From the total stellar mass in IC $1623 \mathrm{~W}\left(3.9 \times 10^{10} \mathrm{M}_{\odot}\right)$, the mass in young, intermediate age and old populations are $1.6 \times 10^{9}$ (4 per cent), $6.4 \times 10^{8}$ ( 1 per cent) and $3.7 \times 10^{10} \mathrm{M}_{\odot}(95$ per cent $)$, respectively. In NGC $6090\left(6.8 \times 10^{10} \mathrm{M}_{\odot}\right)$, the masses in YSP, ISP and OSP are $9.5 \times 10^{8}$ ( 1 per cent), $5.1 \times 10^{9}$ ( 8 per cent $)$ and $6.2 \times 10^{10} \mathrm{M}_{\odot}(91$ per cent), respectively.

In addition, we have compared the mass in clusters, as derived in Section 3, with the mass in YSP derived using the model base CB, which was also used in the photometric study. On the one hand, we found that the mass in star clusters represents only 3 percent and 4 percent of the total stellar mass of IC $1623 \mathrm{~W}$ and NGC 6090 , respectively. On the other hand, in IC $1623 \mathrm{~W}$, the mass in clusters and the mass in YSP from the spectroscopy are comparable to each other within a factor of $\sim 1.3$, in agreement with the idea that the vast majority of stars forms in clusters rather than in isolation (Chandar, Fall \& Whitmore 2015). However, in NGC 6090, the mass in star clusters is a factor $\sim 4$ higher than the mass in SSPs with ages <300 Myr from the spectroscopy. This is within the error expected from the uncertainties in the exact ages of the clusters. From the photometry, we found an upper limit for the ages of $\leq 300 \mathrm{Myr}$, while from the spectroscopy, we found that the young components are in fact younger than $30 \mathrm{Myr}$. The ratio of the mass-to-light ratios for these populations at $\mathrm{Z}_{\odot}$ is approximately $\frac{(M / L)_{300 \mathrm{Myr}}}{(M / L)_{30 \mathrm{Myr}}} \sim 3.6$. Therefore, the age uncertainty in the photometric results could lead to mass uncertainties of up to a factor of $\sim 4$.

\section{IONIZED GAS EMISSION}

The STARLIGHT best fits were subtracted from the observed spectra to obtain 3D cubes with the pure nebular emission-line spectra. These emission spectra contain information about the ionized gas distribution and properties. Using the ratio of $\mathrm{H} \alpha$ to $\mathrm{H} \beta$ intensities, we have determined the ionized gas dust attenuation (Section 5.3), which is necessary to compute the total dereddened $\mathrm{H} \alpha$ luminosity. The $\mathrm{H} \alpha$ luminosity is a commonly used SFR tracer, but can be affected by shock emission. Following Rich et al. (2011), we have analysed the emission-line ratios (Section 5.4) and velocity dispersions (Section 5.5) to confirm which spatial regions are indeed dominated by star formation.

\subsection{Methodology}

We have measured the intensities of some of the most prominent emission lines - [O II] $\lambda 3727, \mathrm{H} \beta,[\mathrm{O} \mathrm{III}] \lambda 5007,\left[\mathrm{O}_{\mathrm{I}}\right] \lambda 6300, \mathrm{H} \alpha$, $[\mathrm{N}$ II] $\lambda 6584$ and [S II] $\lambda \lambda 6716,6731$ - when present in the spectra.

First, a local continuum was estimated using narrow windows located on either side of each line. This local continuum was subtracted to account for small mismatches between the STARLIGHT fit and the actual galaxy continuum. The emission-line intensities in each spectrum were then obtained by fitting Gaussians. Nearby, blended lines (e.g. [N II] and $\mathrm{H} \alpha$ ) were fitted simultaneously using multiple Gaussian profiles. In this way, emission-line intensity maps were created for each individual line.

The statistical uncertainty in the measurement of the line fluxes was calculated from the product of the standard deviation of the adjacent continuum, $\sigma_{\mathrm{c}}$, and the FWHM of the emission line. In order to avoid spurious weak line measurements, we have only considered spaxels where the lines have SNR $>3$.

\subsection{Ionized gas morphology}

IC 1623 W: Fig. 10 shows the observed $\mathrm{H} \alpha$ (left-hand panel) emission flux for IC $1623 \mathrm{~W}$ on a logarithmic scale to enhance the 

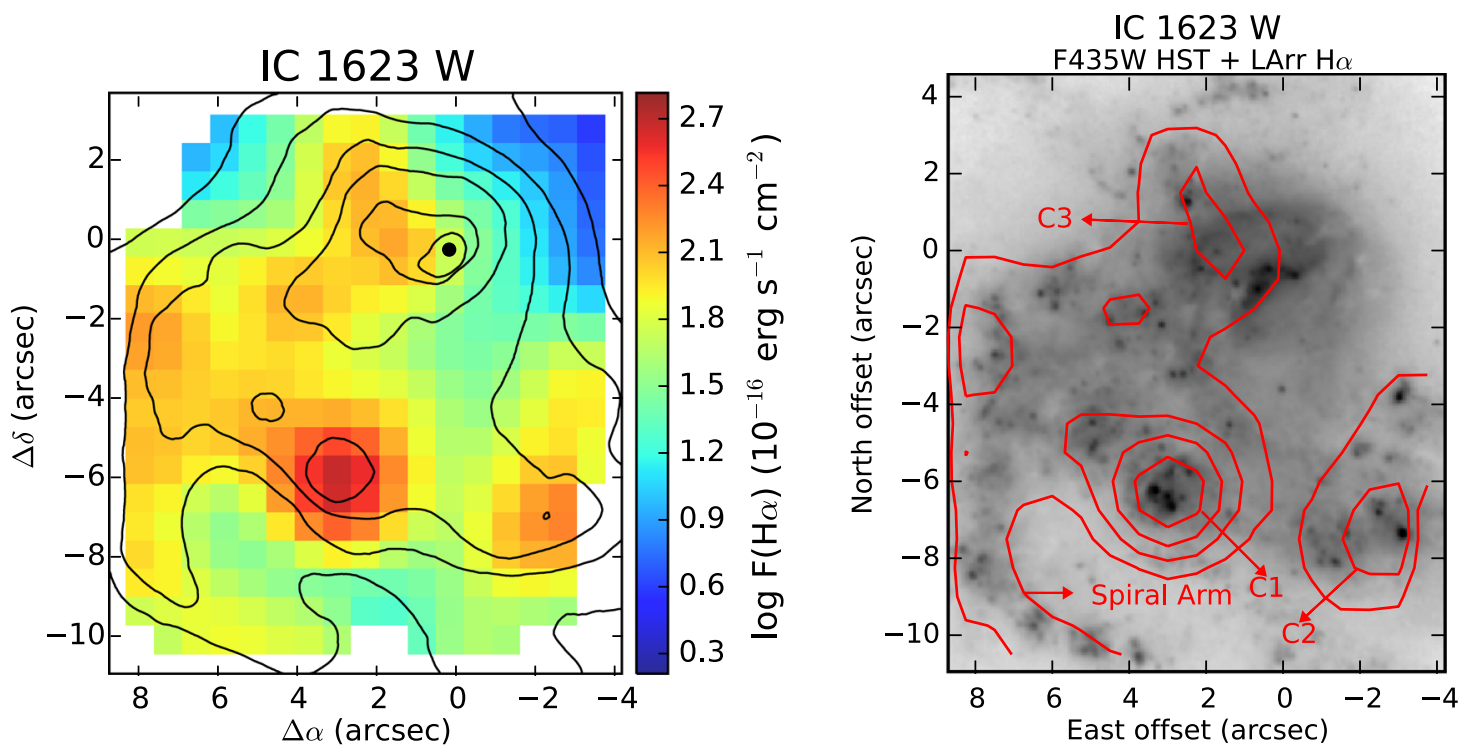

Figure 10. Left-hand panel: $\mathrm{H} \alpha$ emission-line flux in units of $10^{-16} \mathrm{erg} \mathrm{s}^{-1} \mathrm{~cm}^{-2}$ plotted on a logaritmic scale to enhance the contrast, ranging from 0.2 to 2.8. The smoothed HST F435W continuum image is superimposed as contours. Right-hand panel: IC $1623 \mathrm{~W} H S T$ F435W continuum image with LArr H $\alpha$ line emission superimposed as red contours. The distorted spiral arm and SSCs aggregates are labelled in the figure.
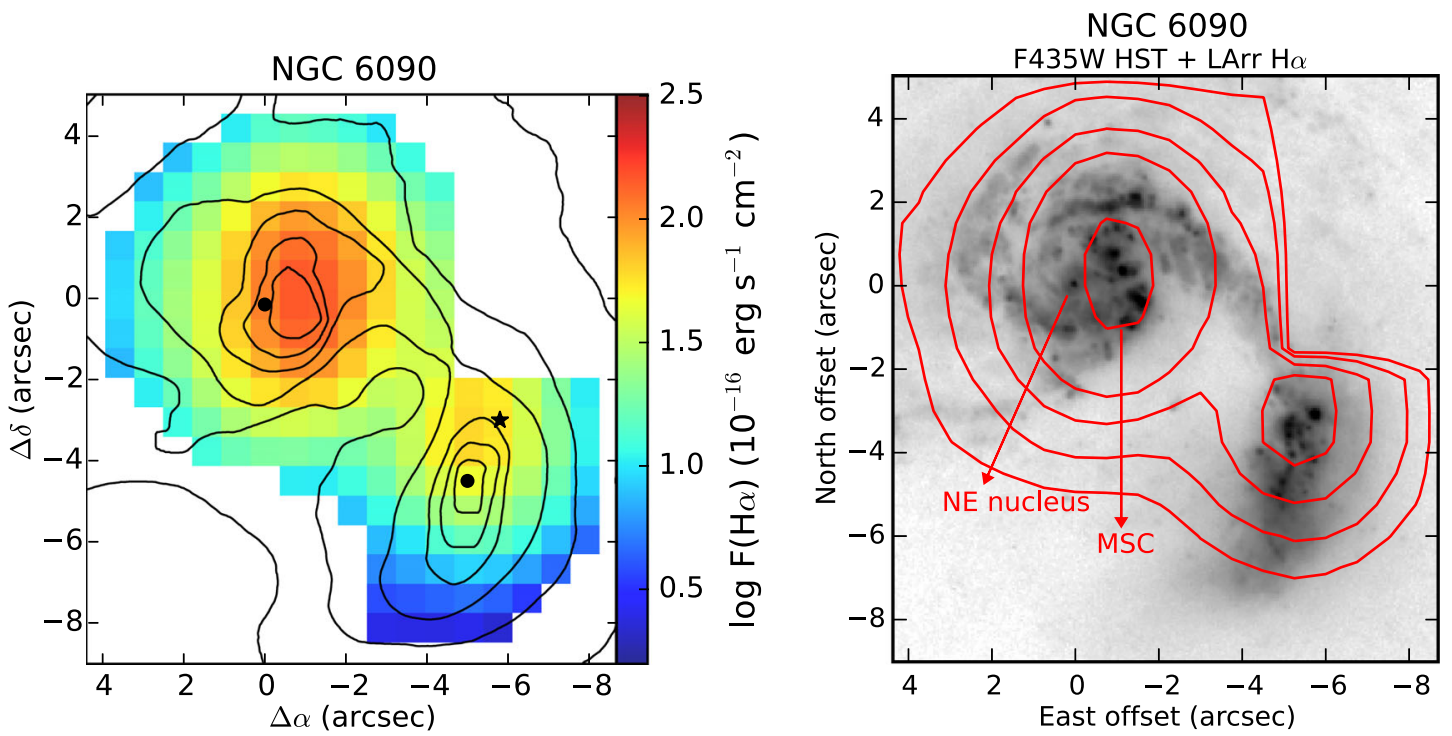

Figure 11. As Fig. 10 except for NGC 6090. In the right-hand panel, the nucleus of the NE galaxy and the region of MSC are labelled.

contrast. As this maps shows, the ionized gas extends across the whole FoV. The brightest regions are those that correspond to aggregates of SSCs and star-forming regions along the distorted spiral arm. To get a better idea of the overall morphology of ionized gas in IC $1623 \mathrm{~W}$, in the right-hand panel of Fig. 10, we have superimposed as red contours the LArr $\mathrm{H} \alpha$ emission over the HST F435W continuum image (grey-scale). We can see that $\mathrm{H} \alpha$ emission is concentrated in the distorted spiral arm and SSCs aggregates (which we have labelled $\mathrm{C} 1$ and $\mathrm{C} 2$ in the image). Furthermore, in the nuclear region, we detect an elongated structure, $\mathrm{C} 3$, which appears to leave or enter the nucleus. Due to its morphology, at first we thought it might be a gas inflow/outflow. Yet, we see in the HST image that this emission has also a stellar counterpart with several SSCs along it. Moreover, we will see in Section 5.4 that the gas ionization is due to star formation in $\mathrm{C} 3$.
NGC 6090: Analogously, in Fig. 11, we show the observed $\mathrm{H} \alpha$ (left-hand panel) map for NGC 6090. The ionized gas distribution is extended across both progenitors in a more uniform and less clumpy way than in IC $1623 \mathrm{~W}$. The NE progenitor is more luminous than the SW one. In the right-hand panel of Fig. 11, we show the HST F435W continuum image of NGC 6090 with the $\mathrm{H} \alpha$ emission superimposed as red contours. In the NE progenitor, the $\mathrm{H} \alpha$ peak is offset to the west of the nucleus, spatially coincident with the MSC region. For the $\mathrm{SW}$ progenitor, $\mathrm{H} \alpha$ peaks in the northern half where most of the star-forming regions accumulate.

\subsection{Ionized gas dust attenuation}

The measured ratio of $\mathrm{H} \alpha$ to $\mathrm{H} \beta$ intensities provides an estimate of the nebular dust attenuation, $A_{V}^{\text {neb }}$, along the line of sight. We 

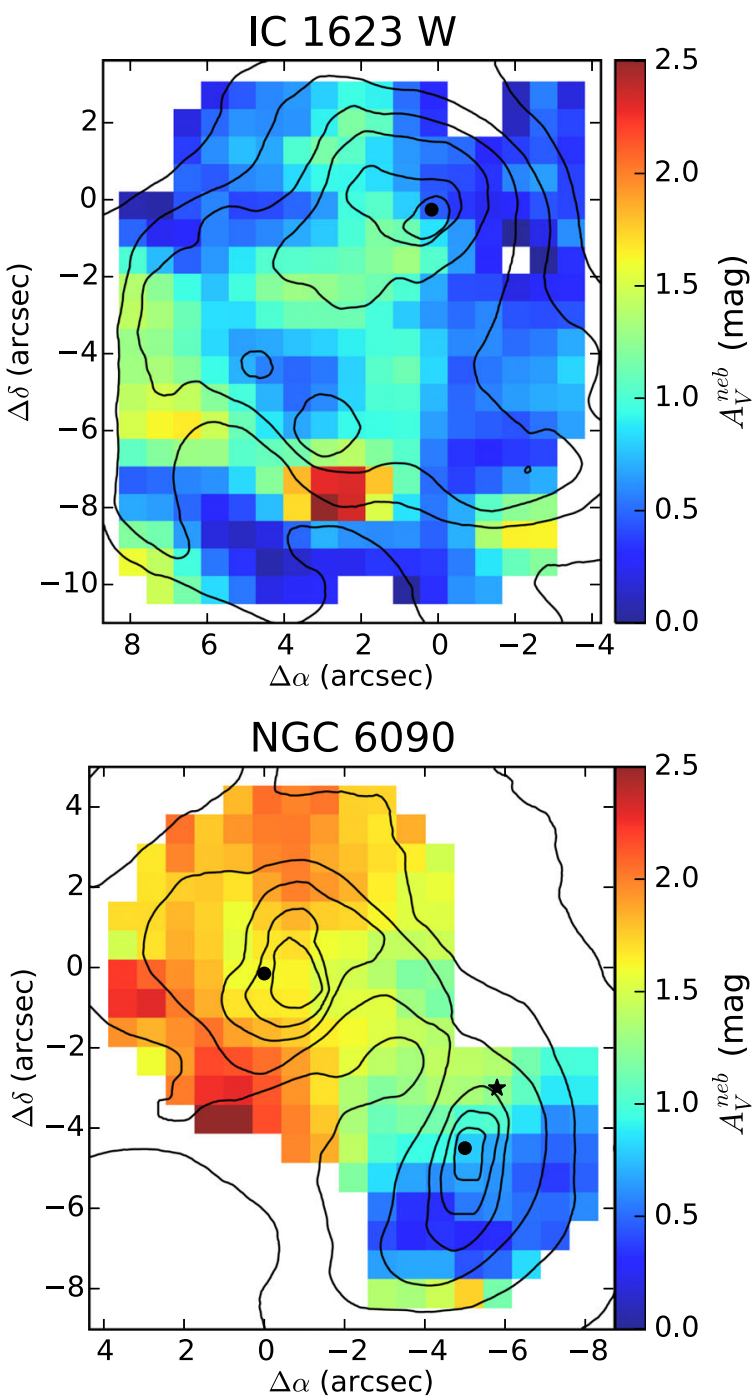

Figure 12. Line attenuation at $5500 \AA$, calculated from the $\mathrm{H} \alpha / \mathrm{H} \beta$ emission-line ratio assuming a Calzetti-like dust attenuation curve for IC $1623 \mathrm{~W}$ (top panels) and NGC 6090 (bottom panels).

assume an intrinsic ratio of $\mathrm{I}(\mathrm{H} \alpha) / \mathrm{I}(\mathrm{H} \beta)=2.86$, valid for case $\mathrm{B}$ recombination with $T=10000 \mathrm{~K}$ and electron density $10^{2} \mathrm{~cm}^{-3}$ (Osterbrock \& Ferland 2006), which are typical of star-forming regions. We have used the Calzetti et al. (2000) reddening function, as it has been shown to work better for starburst galaxies.

In Fig. 12, we show the $A_{V}^{\text {neb }}$ maps for IC $1623 \mathrm{~W}$ (top panel) and NGC 6090 (bottom panel).

In IC $1623 \mathrm{~W}$, the mean nebular extinction is $\sim 0.8$ mag. Following the trend found for the stellar extinction, the regions most affected by extinction are located near areas where Hii regions accumulate. Near $\mathrm{C} 1, A_{V}^{\text {neb }}$ reaches its maximum value of around $2.5 \mathrm{mag}$. The same happens around $\mathrm{C} 2$, with values up to $1.6 \mathrm{mag}$. Also, values between 1.3 and $1.6 \mathrm{mag}$ are found in the distorted spiral arm, and between 1.0 and 1.3 mag to the south-east of the nucleus and along the elongated structure $\mathrm{C} 3$. Everywhere else, the extinction is below $1 \mathrm{mag}$.

In NGC 6090, the average extinction of the NE progenitor is $1.8 \mathrm{mag}$. $A_{V}^{\text {neb }}$ ranges from $1.5 \mathrm{mag}$ in the nucleus of the galaxy, to values around $2 \mathrm{mag}$ in the north, and up to $2.5 \mathrm{mag}$ in a few spaxels in the south. For the SW progenitor, we found an average value around $0.8 \mathrm{mag}$, but it also varies across the galaxy from $0.5 \mathrm{mag}$ in the south to $1 \mathrm{mag}$ in the north. We found an average difference of $\sim 1$ mag between the two progenitors of the system, as also happens with the stellar extinction (see the upper right-hand panel of Fig. 5). In the overlapping bridge region, $A_{V}^{\text {neb }} \sim 1.3-1.4 \mathrm{mag}$.

There is a good spatial correlation between the extended $\mathrm{H} \alpha$ emission and the regions where the YSP are contributing with $\geq 70$ per cent to the total light. As we will see in the next section, the ionization of the warm gas in these early-stage merger LIRGs is indeed due to star formation, as this correlation suggests.

Moreover, we have compared the ratio between the ionized gas extinction to the stellar extinction, using only the spaxels where we are able to determine the ionized gas extinction, i.e. where the SNR of both $\mathrm{H} \alpha$ and $\mathrm{H} \beta$ are above 3 . More than 95 per cent of the spaxels have values of the ratio between 0 and 7 . Values above 7 are considered unreliable, as they correspond to spaxels with $A_{V}^{\text {stars }}$ below the uncertainty with which we can determine it (of $\sim 0.1 \mathrm{mag}$; CF2013). For IC 1623, we found $\left\langle\frac{A_{V}^{\text {neb }}}{A_{V}^{\text {stars }}}\right\rangle=2.2 \pm 1.4$ (median and standard deviation), while for NGC 6090, $\left\langle\frac{A_{V}^{\text {neb }}}{A_{V}^{\text {stars }}}\right\rangle=1.4 \pm 0.8$. Analysing UV and optical spectra of 39 starburst galaxies, Calzetti, Kinney \& Storchi-Bergmann (1994) found $\left\langle\frac{A_{V}^{\text {neb }}}{A_{V}^{\text {tsars }}}\right\rangle=2.0 \pm 0.4$, which is compatible with the results from our early-stage merger LIRGs. The fact that in starbursts and interacting galaxies, $A_{V}^{\text {neb }}$ is larger than $A_{V}^{\text {stars }}$ is attributed to the fact that emission-line sources are preferentially located within dusty birth clouds, whereas the stellar light mainly samples the diffuse dust in the ISM. Kreckel et al. (2013) also find a similar result analysing IFS data from eight nearby galaxies, but noting a more complex behaviour of the nebular to stellar extinction ratio, which is sensitive to the $\mathrm{H} \alpha$ flux.

\subsection{Ionisation mechanisms: star formation and shocks}

Diagnostic diagrams comparing emission-line flux ratios are the most widely used method for identifying the nature of the dominant ionization mechanisms in galaxies. Fig. 13 presents two such diagrams: $\left[\mathrm{N}_{\mathrm{II}}\right] \lambda 6584 / \mathrm{H} \alpha$ versus $\left[\mathrm{O}_{\mathrm{III}}\right] \lambda 5007 / \mathrm{H} \beta$ (left-hand panels, henceforth the 'BPT diagram', after Baldwin, Phillips \& Terlevich 1981), and [O I] $\lambda 6300 / \mathrm{H} \alpha$ versus [O III] $\lambda 5007 / \mathrm{H} \beta$ (middle panel, following Veilleux \& Osterbrock 1987) for both IC 1623 (top panels) and NGC 6090 (bottom panels). Panels on the right show the $\mathrm{H} \alpha$ velocity dispersion $\left(\sigma_{\mathrm{H} \alpha}\right)$ maps.

Solid lines in both diagnostic diagrams mark the 'maximum starburst lines' obtained by K01 by means of photoionization models of synthetic starbursts. These lines are often interpreted as delimiting the region occupied by 'pure AGN', even though this is not what they were designed for (see the discussion in Cid Fernandes et al. 2011). The dotted line in the left-hand panels marks the frontier semi-empirically drawn by K03 to separate SDSS starforming galaxies from those hosting an AGN. Systems between these two lines are often called 'composite', a nomenclature that betrays the traditional interpretation of such systems as a mixture of star-forming and AGN.

None of the spaxels in either IC 1623 or NGC 6090 lies above the K01 line in the BPT diagram (left-hand panels) and only a few do so in the $\left[\mathrm{O}_{\mathrm{I}}\right] / \mathrm{H} \alpha$ one (middle panels), indicating that AGN, even if present, contribute little to the emission lines. In fact, most of the points lie below the K03 line, confirming that photoionization by young stars is the main mechanism of line production in these sources. Black stars in these panels mark the line ratios of the spatially integrated spectra, and their position in the diagnostic 

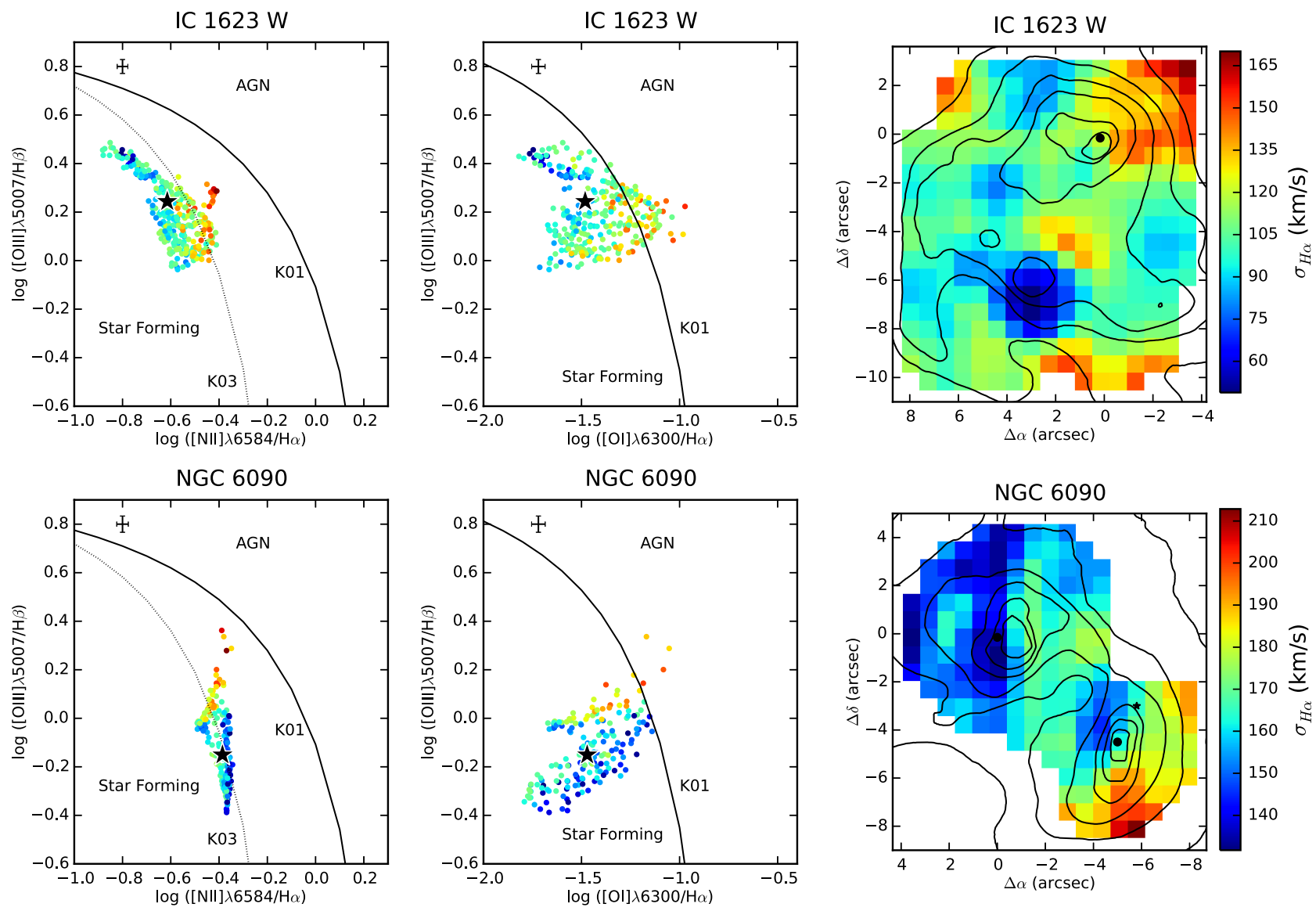

Figure 13. [ $\left.\mathrm{N}_{\mathrm{II}}\right] \lambda 6584 / \mathrm{H} \alpha$ versus [O $\left.\mathrm{III}\right] \lambda 5007 / \mathrm{H} \beta$ (left-hand panels), and $\left[\mathrm{O}_{\mathrm{I}}\right] \lambda 6300 / \mathrm{H} \alpha$ versus $\left[\mathrm{O}_{\mathrm{III}}\right] \lambda 5007 / \mathrm{H} \beta$ (middle panels) line ratio diagnostic diagrams, and $\mathrm{H} \alpha$-based velocity dispersion maps (right-hand panels) for IC $1623 \mathrm{~W}$ (top panels) and NGC 6090 (bottom panels). Spaxels are colour coded according to the velocity dispersion. The ratios measured in the integrated spectra are shown with black stars. Solid lines in both diagnostic diagrams mark the 'maximum starburst lines' obtained by Kewley et al. (2001) (hereafter K01) by means of photoionization models of synthetic starbursts, and the dotted line in the left-hand panels marks the pure SDSS star-forming galaxies frontier semi-empirically drawn by Kauffmann et al. (2003) (hereafter K03). The average error bars are shown in the top left-hand corners. The velocity dispersions in the right-hand panels have been corrected for the instrumental width $\left(\sigma_{\text {inst }} \sim 140 \mathrm{~km} \mathrm{~s}^{-1}\right)$.

diagrams further confirms the dominance of star formation as the main ionization source.

Interestingly, however, in both cases, a significant number of spaxels occupy the intermediate zone between K03 and K01 in BPT-coordinates. Moreover, the colour coding makes it evident to the eye that the points that depart the most from the star-forming sequence are those with high $\sigma_{\mathrm{H} \alpha}$ (yellow-red colours). In IC 1623, these large gas velocity dispersion regions are mostly located towards the NW of the $\sigma_{\mathrm{H} \alpha}$ map (top right-hand panel), while in NGC 6090 (bottom right-hand panel), they are concentrated in the south of NGC $6090 \mathrm{SW}$.

These are signs of tidally induced shocks, associated with the interaction process, contributing to the ionization of the gas (Colina, Arribas \& Monreal-Ibero 2005). Extended shock ionization has been previously reported in local U/LIRGs, taking advantage of IFS studies (Monreal-Ibero, Arribas \& Colina 2006; Rich et al. 2011; Rich, Kewley \& Dopita 2014). In all these cases, shock ionization exhibits characteristics of extended low-ionization nuclear emission-line region (LINER)-like emission with broadened line profiles. In the case of our IFS data for IC $1623 \mathrm{~W}$ and NGC 6090, we find broadened line profiles falling in the so-called composite region. The 'composite' systems are conventionally associated with a superposition of normal $\mathrm{H}_{\text {II }}$ regions and the narrow-line region of
AGN. However, the location of these shocked regions in BPT coordinates, together with the spatial coherence of the line ratios and kinematical data revealed in our IFS, allow us to circumvent these limitations and correctly identify the gas ionization mechanism in our early-stage merger systems.

It is further useful to note that the misidentification of shocked regions with a mixture of star formation and AGN ionization is not the only caveat in a naive reading of our diagnostic diagrams. In the case of NGC 6090 , some low $\sigma_{\mathrm{H} \alpha}$ spaxels populate the zone between the $\mathrm{K} 03$ and $\mathrm{K} 01$ demarkation lines (blue points in the bottom left-hand panel of Fig. 13). Their off-nuclear locations and closeness to the $\mathrm{K} 03$ line argue in favour of them being pure $\mathrm{H}$ II regions, and not 'star-forming + AGN composite' systems, which their nominal locations in the BPT diagram would indicate. This caveat has been noted before by Sánchez et al. (2015), who find that some $\sim 14$ per cent of the bona fide $\mathrm{H}$ II regions in the CALIFA sample lie in the (ill-named) 'composite' region.

\subsection{Ionized gas kinematics: velocity dispersion}

We trace the gas kinematics of NGC 6090 and IC $1623 \mathrm{~W}$ using the results of Gaussian fits to their emission lines. To obtain the final 
velocity dispersion, we have subtracted the instrumental resolution $\left(\sigma_{\text {inst }}\right)$ in quadrature, where $\sigma_{\text {inst }}$ at $\mathrm{H} \alpha$ is $\sim 140 \mathrm{~km} \mathrm{~s}^{-1}$.

In the right-hand panel of Fig. 13, we show the ionized gas velocity dispersion maps for IC 1623 W (top panel) and NGC 6090 (bottom panel).

In IC $1623 \mathrm{~W}$, the velocity dispersion is low in the star-forming regions and in the distorted spiral arms, with $\sigma_{\mathrm{H} \alpha}<120 \mathrm{~km} \mathrm{~s}^{-1}$. However, $\sigma_{\mathrm{H} \alpha}>120 \mathrm{~km} \mathrm{~s}^{-1}$ in several outermost spaxels, with a region to the NW of the nucleus where $\sigma_{\mathrm{H} \alpha}$ reaches up to $170 \mathrm{~km} \mathrm{~s}^{-1}$.

In NGC 6090, there exists a gradual increase in the velocity dispersion from $\sim 140-150 \mathrm{~km} \mathrm{~s}^{-1}$ to the east side of NE progenitor, to $170 \mathrm{~km} \mathrm{~s}^{-1}$ in the bridge between progenitors, and up to $190 \mathrm{~km} \mathrm{~s}^{-1}$ to the SW of the SW progenitor.

We interpret the high-velocity dispersion regions in these earlystage mergers as a consequence of shocks producing a broadening of the emission lines. Moreover, in Section 5.4, we saw that there exists a correspondence between these regions and 'composite' excitation conditions in the BPT diagram. IFS data for IC 1623 were previously analysed by Rich et al. (2011), who found widespread shocks (LINER-like ionization) in the outskirts of the system, beyond the region traced by our data. In the region in common (the core of IC $1623 \mathrm{~W}$ ), our results are in total agreement with them, finding similar values of the lines ratios $\left[\mathrm{N}_{\mathrm{II}}\right] / \mathrm{H} \alpha \sim-0.9$ to -0.4 , $[\mathrm{O}$ I $] / \mathrm{H} \alpha \sim-1.7$ to -1.0 , consistent with star formation ionization. While Rich et al. (2011) are able to further decouple the contribution arising from shocks and star formation, we cannot follow a similar approach due to our lower spectral resolution $\left(140 \mathrm{~km} \mathrm{~s}^{-1}\right.$ versus $40 \mathrm{~km} \mathrm{~s}^{-1}$ in $\mathrm{H} \alpha$ ).

\subsection{Gas metallicities}

We estimate the nebular metallicity of the star-forming regions using the $\mathrm{O} 3 \mathrm{~N} 2 \equiv \log \frac{[\mathrm{O} \mathrm{III}] \lambda 5007 / \mathrm{H} \beta}{[\mathrm{NII}] / \mathrm{H} \alpha}$ index, as calibrated by Marino et al. (2013). To exclude shocked regions from this analysis in an approximate way we remove spaxels with $\sigma_{\mathrm{H} \alpha}>130 \mathrm{~km} \mathrm{~s}^{-1}$ in IC $1623 \mathrm{~W}$ and $\sigma_{\mathrm{H} \alpha}>185 \mathrm{~km} \mathrm{~s}^{-1}$ in NGC 6090 , corresponding to the points marked in yellow-red in Fig 13 .

The resulting values of $12+\log (\mathrm{O} / \mathrm{H})$ range from 8.20 to 8.40 in IC $1623 \mathrm{~W}$, and from 8.40 to 8.52 in NGC 6090. For a solar value of $12+\log (\mathrm{O} / \mathrm{H})=8.69$ (Asplund et al. 2009), these values correspond to $\sim 0.4$ and 0.6 solar (on average) for IC $1623 \mathrm{~W}$ and NGC 6090, respectively.

Also, in Fig. 14, we compare the nebular oxygen metallicity in red, with the oxygen abundance of the Milky Way (MW) in blue, obtained using the calibration by Afflerbach et al. (1997), and with the stellar metallicities in black. The early-stage mergers have lower central oxygen metallicities in comparison with the MW, and flatter profiles. The nebular and stellar metallicities are similar, both in IC $1623 \mathrm{~W}$ and NGC 6090, but the stellar metallicities show slightly more positive gradients, while the nebular profiles are flat. However, a larger sample and more spatial coverage would be necessary to assess these results in a statistically significant way. In Section 6.4, our results are discussed in the context of the literature.

\section{DISCUSSION}

In this section, we discuss our main findings in the context of the merger-driven evolution of galaxies. The observational characterization of star formation in mergers at different stages is necessary to test the validity and put constraints on merger simulations. Merging LIRGs, such as the ones presented in this paper, are unique laboratories to study this in detail. First, we calculate and compare
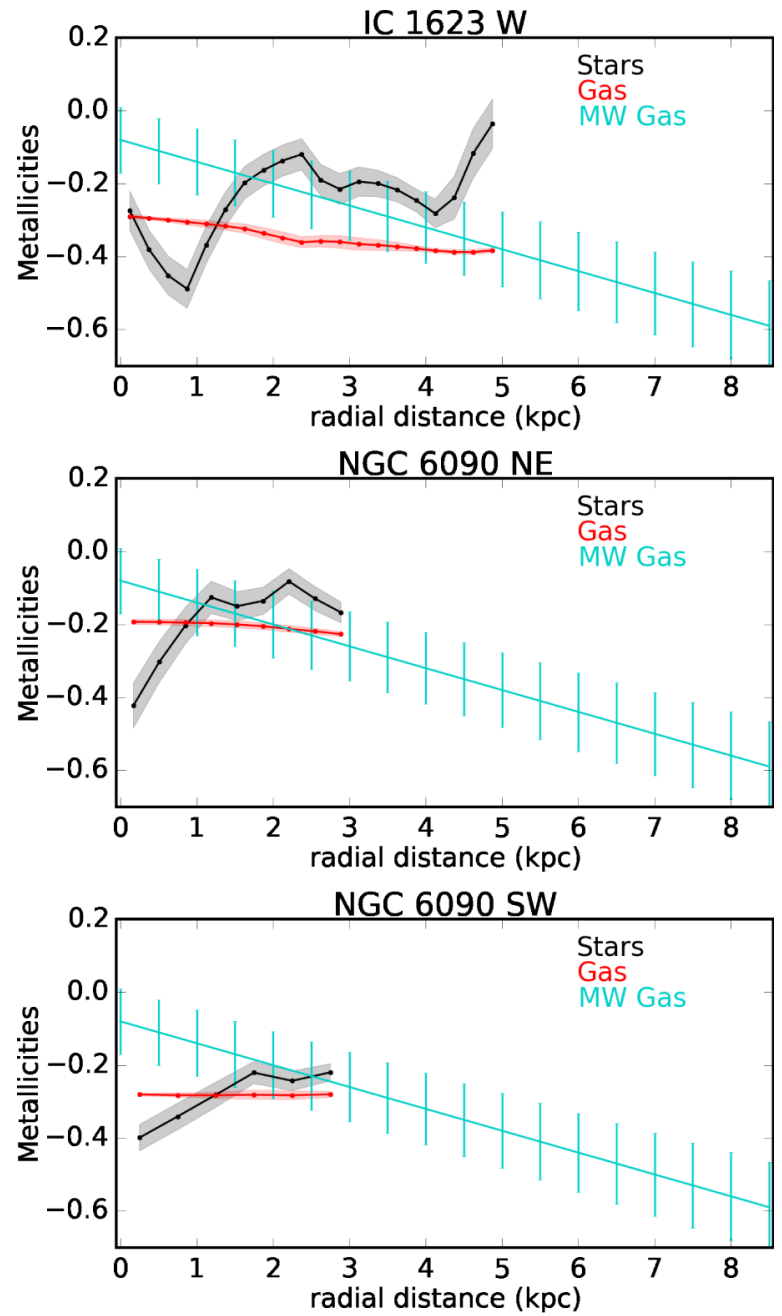

Figure 14. Oxygen metallicity profiles for our early-stage mergers in red, compared to the stellar metallicities in black, and the Milky Way oxygen metallicity in blue, the latter assuming the Afflerbach, Churchwell \& Werner (1997) calibration. For comparison, all values are in log-solar units, and the spatial scale is in kpc.

the current SFR of IC $1623 \mathrm{~W}$ and NGC 6090, using the spectral synthesis, $\mathrm{H}_{\alpha}$ and MIR data.

\subsection{Multiwavelength SFRs}

We explore two ways to estimate SFRs from our IFS data. First, we compute the SFR from the dereddened $\mathrm{H} \alpha$ luminosity considering that shocks are not important, i.e. assuming that the gas is photoionized by massive stars only. We use the conversion to SFR provided by Lacerda (private communication), calculated for the average metallicity of our galaxies $\sim 0.7 \mathrm{Z}_{\odot}$ and for Bruzual \& Charlot (2003) models. The calibration with a Salpeter IMF is very similar to the standard calibration of Kennicutt (1998), calculated for solar metallicity models.

Secondly, we use our stellar continuum decomposition to estimate the SFR by summing all the stellar mass formed since a lookback time $t_{\mathrm{SF}}$ and performing a mass-over-time average, following equation 11 in CF2013, which gives the mean SFR since $t=t_{\mathrm{SF}}$. One can tune $t_{\mathrm{SF}}$ to reach different depths in the past. For comparison purposes, we estimate it for $t_{\mathrm{SF}}=30 \mathrm{Myr}$ (González Delgado et al. 2016; Asari et al. 2007), which is of the same order 
of the lifetime of the ionizing populations also emitting in $\mathrm{H} \alpha$, i.e. $\mathrm{O}$ and early B stars.

Additionally, we have used a hybrid calibration, including the Spitzer MIPS $24 \mu \mathrm{m}$ flux to check whether there is any star formation completely obscured from view in the optical, which is not accounted for by the Balmer decrement attenuation correction. We have followed the calibration given by Kennicutt et al. (2009).

The three different approaches lead to very similar SFR results, with an average and dispersion of $23 \pm 4 \mathrm{M}_{\odot} \mathrm{yr}^{-1}$ for IC $1623 \mathrm{~W}$, and $32 \pm 4 \mathrm{M}_{\odot} \mathrm{yr}^{-1}$ for NGC 6090, for Salpeter IMF, and a factor of 1.6-1.7 lower with Chabrier IMF models. The consistency between the hybrid calibration including MIR and the optical ones indicates that for IC $1623 \mathrm{~W}$ and NGC 6090, there is no substantial star formation that is not accounted for by the optical data.

\subsection{Merger-induced star formation in mergers}

\subsubsection{Global enhancement of star formation}

In both IC $1623 \mathrm{~W}$ and NGC 6090, we find that the SFR is increased with respect to the so-called main-sequence star-forming galaxies (MSSF; Renzini \& Peng 2015). Using the González Delgado et al. (2016) calibration for the MSSF in the same mass range as our earlystage mergers $(\sim \mathrm{Sbc})$, we obtain values of $\sim 3.0-4.4 \mathrm{M}_{\odot} \mathrm{yr}^{-1}$, a factor 6-9 lower than the SFRs of our two early-stage mergers. This increase is larger than the predictions from classical merger simulations by Di Matteo et al. (2008), who found that boosts in the SFR greater than 5 are rare, but still possible in about 15 per cent of major galaxy interactions and mergers. We suggest that U/LIRG population could belong to this 15 percent in the sense that star formation in them is 'caught in the act'.

As the SFR depends on the total amount of mass, we have also calculated the average specific star formation rate, $\mathrm{SSFR}=\mathrm{SFR} / M_{\star}$, which is sSFR $\sim 0.60 \mathrm{Gyr}^{-1}$ for IC $1623 \mathrm{~W}$, and $0.47 \mathrm{Gyr}^{-1}$ for NGC 6090; these are significantly larger (a factor of 5-6) than the sSFR of the Sbc/Sc control galaxies measured at $1 \mathrm{HLR}\left(\sim 0.1 \mathrm{Gyr}^{-1}\right){ }^{4}$ Therefore, the star formation increase in our two early-stage mergers is still significant even after normalization by the total stellar mass. This is expected given that the control samples have been selected in the same mass range as early-stage mergers. Moreover, this sSFR can be interpreted as the inverse of a time-scale for the star formation $(\tau)$. We find that $\tau$ is much shorter in our early-stage mergers $(\sim 2.0 \mathrm{Gyr})$ than in Sbc/Sc galaxies of similar mass $(\sim 10 \mathrm{Gyr})$. That is, the same stellar mass has been built much faster in the early-stage mergers than in the control samples.

\section{2 .2 IC 1623}

From the IFS and star cluster photometry, we find that mergerinduced star formation in IC 1623 is both widespread (out to 1.5 HLR) and recent, with an average light-weighted age of $\sim 50 \mathrm{Myr}$ in IC $1623 \mathrm{~W}$. The centre of IC $1623 \mathrm{~W}(<0.5 \mathrm{HLR})$ is older, $\sim 300 \mathrm{Myr}$, than the surrounding area, $\sim 30 \mathrm{Myr}$ (Fig. 6), due to the presence of a significant contribution of ISP (Fig. 8) probably induced in a previous stage of the merger. However, the light is dominated everywhere by YSP, with contributions in the range of 50-90 per cent across IC $1623 \mathrm{~W}$ compared with $10-20$ per cent in the $\mathrm{Sbc} / \mathrm{Sc}$ control galaxies. The extinction in this progenitor is low

\footnotetext{
${ }^{4}$ This comparison can be made because we have found that the galaxy-wide average stellar population properties are well represented by their values measured at 1 HLR González Delgado et al. (2015b).
}

$\sim 0.2 \mathrm{mag}$, and has a flat radial profile in comparison with $\mathrm{Sbc} / \mathrm{Sc}$ control spirals (Fig. 5). However, we note that IC $1623 \mathrm{E}$ is affected by much higher levels of extinction (up to 2-6 mag) according to the star cluster photometry (Figs 3 and A2). IC $1623 \mathrm{~W}$ has a mean stellar metallicity of $\sim 0.6 \mathrm{Z}_{\odot}$, with a flat or slightly positive radial profile (Fig. 7). The nebular metallicity profile is also flatter than in non-interacting spiral galaxies and consistent with the existence of gas inflows of metal-poor gas to the centre of the system, as explained below in Section 6.4 .

\subsubsection{NGC 6090}

As for IC 1623, the merger-induced star formation in NGC 6090 is widespread, as indicated by the star clusters detected in the HST images (Fig. 3), and recent, with an average light-weighted age from the IFS of $\sim 80 \mathrm{Myr}$, with both progenitors, NGC $6090 \mathrm{NE}$ and NGC 6090 SW, having similar ages (Fig. 6). As in IC 1623 W, the age profiles are significantly flatter than in Sbc/Sc galaxies, in agreement with a general rejuvenation of the progenitor galaxies due to merger-induced star formation. The light is dominated by YSP in both progenitors, with contributions $\sim 70$ per cent (Fig. 8). There exist a difference of $\sim 1$ mag between the stellar extinctions of the two progenitors, with an average of $1.3 \mathrm{mag}$ in NGC $6090 \mathrm{NE}$, and $0.5 \mathrm{mag}$ in NGC $6090 \mathrm{SW}$ (Fig. 5), and whose radial profiles are flat in comparison with $\mathrm{Sbc} / \mathrm{Sc}$ control spirals. As in IC $1623 \mathrm{~W}$, NGC 6090 has a mean stellar metallicity of $\sim 0.6 \mathrm{Z}_{\odot}$, with a flat or slightly positive radial profile (Fig. 7), and a flat nebular metallicity profile, also in agreement with the gas inflow scenario.

\subsubsection{Evolutionary scheme}

Based on our results, we propose an evolutionary scheme in which the gas components of the progenitors react promptly to the merger process, probably during the first contact (stage II in Veilleux et al. 2002 classification), where the discs overlap but strong bars and tidal tails have not yet formed. During this stage the gas starts inflowing to the dynamical centre of the system, and can trigger some star formation. The ISP in IC $1623 \mathrm{~W}$ nucleus ( $300 \mathrm{Myr}$ ) could be a consequence of this, and we note that the star formation triggered during the first contact stage is still not extended, but concentrated in the centre. The early-stage merger (III in Veilleux et al. 2002) starts after the first pericentre passage (in our systems, in the last $<50$ $100 \mathrm{Myr}$ ), where most of the extended star formation is due to the fragmentation of gas clouds produced by the increase of the supersonic turbulence of the ISM, as predicted from high-resolution models in the literature (Teyssier et al. 2010; Hopkins et al. 2013; Renaud et al. 2015). Therefore, at this stage, the star formation is mainly in the form of star clusters. We found that the mass in the star clusters is similar to that of the 140-Myr age stellar populations derived from the IFS data (within factors of 1.3 and $\sim 4$ for IC $1623 \mathrm{~W}$ and NGC 6090, respectively.) Spatially extended star formation has also been previously observed in other early-stage merger systems (Hattori et al. 2004; Wang et al. 2004; Elmegreen et al. 2006; García-Marín et al. 2009; Arribas et al. 2012). The central nebular metallicities are already diluted at the early merger stage, at least in some cases.

\subsection{Ionization mechanism}

In both IC $1623 \mathrm{~W}$ and NGC 6090, the dominant ionization mechanism is photoionization by young massive stars. For a few spaxels, we cannot discard composite photoionization by hot stars and 
shock ionization, given also the high velocity dispersion in the north-west of IC $1623 \mathrm{~W}$ and to the south of NGC $6090 \mathrm{SW}$. This result is in agreement with previous results from the literature (Rich et al. 2011, 2014).

\subsection{Nebular metallicity gradients}

Finally, in the literature, there exists some controversy about the evolution of the gas metallicity in mergers, with some studies finding lower central metallicity in mergers in comparison to control galaxies, and a flattening of the metallicity profiles, possibly related with gas inflows (Kewley et al. 2010; Rich et al. 2012; Guo et al. 2016), and others finding a similar central metallicity in mergers and control samples, with a decrease compared to the control sample objects at the large radii. This suggests that other processes such as stellar feedback can contribute to the metal enrichment in interacting galaxies (Barrera-Ballesteros et al. 2015).

Nebular metallicities estimated with the O3N2 method are $\sim 0.4$ and 0.6 solar in IC $1623 \mathrm{~W}$ and NGC 6090, respectively. In Section 5.6, we showed the oxygen metallicity profiles of our earlystage mergers. In particular, for IC $1623 \mathrm{~W}$, we found a gradient of $-0.020 \pm 0.013 \mathrm{dex} \mathrm{kpc}^{-1}$, which is in agreement with the gradient determined by Rich et al. (2012) using Kewley \& Dopita (2002) calibration ( $\left.-0.016 \mathrm{dex} \mathrm{kpc}^{-1}\right)$. Rich et al. (2012) also compute a set of merger simulations to trace the oxygen metallicity variation. For close pairs (stage 2), they found a metallicity gradient of $-0.025 \pm 0.015 \mathrm{dex} \mathrm{kpc}^{-1}$, consistent with that found by us in IC $1623 \mathrm{~W}$. In NGC 6090, we find even flatter profiles of $-0.012 \pm 0.013 \mathrm{dex} \mathrm{kpc}^{-1}$ in the NE progenitor, and $0.000 \pm 0.014$ dex $\mathrm{kpc}^{-1}$ in the SW progenitor, but still within the observational range of close pairs in the Rich et al. (2012) study. All of them are flatter than the MW gradient $\left(-0.060 \pm 0.035 \mathrm{dex} \mathrm{kpc}^{-1}\right)$, and the control sample of isolated star-forming spirals of Rupke, Kewley \& Chien (2010) $\left(-0.041 \pm 0.009 \mathrm{dex} \mathrm{kpc}^{-1}\right)$, in agreement with the gas inflow scenario as the main cause of the dilution.

\section{SUMMARY AND CONCLUSIONS}

Through IFS PMAS LArr data and multiwavelength HST imaging, and with the STARLIGHT full spectral fitting code, in this paper, we characterize the star cluster properties, stellar populations, ionized gas properties (ionization mechanism and kinematics) and SFRs in the early-stage mergers LIRGs IC 1623 W and NGC 6090. Earlystage merger results have been compared with control Sbc and Sc galaxies from CALIFA (GD2015) in the same mass range. Our main results are as follows.

(i) From the IFS and star cluster photometry, we find that mergerinduced star formation in these two LIRGs is widespread (out to 1.5 HLR) and recent, as revealed by the young light-weighted ages (50-80 Myr versus $\sim 1 \mathrm{Gyr}$ in $\mathrm{Sbc} / \mathrm{Sc}$ control galaxies) and the high contributions to light of YSP (50-90 per cent versus 10 20 per cent in $\mathrm{Sbc} / \mathrm{Sc}$ galaxies). These results are in agreement with high-resolution merger simulations in the literature.

(ii) The $A_{V}$ profile in the inner 1 HLR of these two early stage mergers is flat in comparison with the negative gradient in $\mathrm{Sbc} / \mathrm{Sc}$ control spirals.

(iii) The age profiles are significantly flatter than in Sbc/Sc galaxies, in agreement with a general rejuvenation of the progenitor galaxies due to merger-induced star formation.

(iv) The average stellar mass-weighted metallicities at 1 HLR are $\sim 0.6 \mathrm{Z}_{\odot}$, similar to Sbc galaxies. Although with a large scat- ter, the stellar metallicity profiles are comparatively flat or slightly positive as compared to $\mathrm{Sbc} / \mathrm{Sc}$ galaxies.

(v) Nebular abundances estimated with the O3N2 method are $\sim 0.4$ and 0.6 solar in IC $1623 \mathrm{~W}$ and NGC 6090, respectively.

(vi) The nebular abundance profiles are flatter $(-0.020 \pm 0.013$ dex kpc ${ }^{-1}$ gradient in IC $1623 \mathrm{~W},-0.012 \pm 0.013 \mathrm{dex} \mathrm{kpc}^{-1}$ in NGC $6090 \mathrm{NE}$ and $0.000 \pm 0.014 \mathrm{dex} \mathrm{kpc}^{-1}$ in NGC $6090 \mathrm{SW}$ ) than the ones in the MW and in a sample of isolated star-forming spirals, in agreement with the scenario where gas inflows are responsible of the dilution.

(vii) From the $[\mathrm{N}$ II] $\lambda 6583 / \mathrm{H} \alpha$ versus $[\mathrm{O}$ III] $\lambda 5007 / \mathrm{H} \beta$, and [O I] $\lambda 6300 / \mathrm{H} \alpha$ versus $[\mathrm{O}$ III $] \lambda 5007 / \mathrm{H} \beta$ diagnostic diagrams, we find that both IC $1623 \mathrm{~W}$ and NGC 6090 are dominated by star formation in the region mapped in this work.

(viii) In some regions, we cannot discard a mix of photoionization by hot stars and by shocks, given the high velocity dispersion to the south of NGC 6090 SW, and to the northwest of IC $1623 \mathrm{~W}$, consistent with previous results from the literature.

(ix) We obtained several estimations of the current SFR using the spectral synthesis [SFR(30 Myr)], the dereddened $\mathrm{H} \alpha$ luminosity $[\operatorname{SFR}(\mathrm{H} \alpha)]$ and a hybrid calibration using the observed $\mathrm{H} \alpha$ luminosity and MIR $24 \mu \mathrm{m}$ luminosity. All of them lead to very similar values of the SFR, with an average of $23 \pm 4 \mathrm{M}_{\odot} \mathrm{yr}^{-1}$ for IC $1623 \mathrm{~W}$, and $32 \pm 4 \mathrm{M}_{\odot} \mathrm{yr}^{-1}$ for NGC 6090. From the good consistency between the different estimations, we conclude that for IC $1623 \mathrm{~W}$ and NGC 6090, there is no substantial star formation that is not accounted for by the optical data.

(x) The average SFR in our early-stage mergers is enhanced by factors of 6-9 with respect to main-sequence Sbc star-forming galaxies. This is slightly above the predictions from classical merger simulations, which indicate that SFR enhancement factors greater than 5 are rare, but still possible in about 15 per cent of major galaxy interactions and mergers. We suggest that U/LIRGs could belong to this 15 per cent in the sense that star formation in them is caught in the act.

(xi) The sSFR in IC $1623 \mathrm{~W}\left(\sim 0.60 \mathrm{Gyr}^{-1}\right)$ and NGC 6090 $\left(\sim 0.47 \mathrm{Gyr}^{-1}\right)$ is also enhanced by factors of $5-6$ with respect to Sbc/Sc galaxies $\left(\sim 0.1 \mathrm{Gyr}^{-1}\right)$.

(xii) Interpreting the sSFR as the inverse of a time-scale for the $\mathrm{SF}$, our results indicate that at their present SFR, early-stage mergers build their stellar mass much faster $(\sim 2 \mathrm{Gyr})$ than $\mathrm{Sbc/Sc}$ control galaxies $(\sim 10 \mathrm{Gyr})$.

(xiii) Our results are consistent with an evolutionary scheme where some star formation can be initially triggered in the central regions during the first contact stage, followed by extended star formation after the first pericentre passage (early stage mergers).

\section{ACKNOWLEDGEMENTS}

CALIFA is the first legacy survey carried out at Calar Alto. The CALIFA collaboration would like to thank the Instituto de Astrofísica de Andalucía-Centro Superior de Investigaciones Científicas (IAA-CSIC) and Max Planck Institut für AstronomieMax Planck Gesellschaft (MPIA-MPG) as major partners of the observatory, and CAHA itself, for the unique access to telescope time and support in manpower and infrastructures. We also thank the CAHA staff for the dedication to this project. The authors acknowledge the support from the Spanish Ministerio de Economía y Competitividad, through projects AYA2014-57490-P, AYA201015081, and Junta de Andalucía FQ1580. ALdA, EADL and RCF thank the hospitality of the IAA and the support of Coordenação 
de aperfeiçoamento de pessoal de nivel superior (CAPES) and Conselho Nacional de Pesquisa (CNPq). RGD acknowledges the support of $\mathrm{CNPq}$ (Brazil) through Programa Ciência sem Fronteiras (401452/2012-3). Also based on observations made with the NASA/ESA Hubble Space Telescope, and obtained from the Hubble Legacy Archive, which is a collaboration between the Space Telescope Science Institute (STScI/NASA), the Space Telescope European Coordinating Facility (ST-ECF/ESA) and the Canadian Astronomy Data Centre (CADC/NRC/CSA).

\section{REFERENCES}

Afflerbach A., Churchwell E., Werner M. W., 1997, ApJ, 478, 190

Alvensleben U. F., 2004, in Lamers H. J. G. L. M., Smith L. J., Nota A., eds, ASP Conf. Ser. Vol. 322, The Formation and Evolution of Massive Young Star Clusters. Astron. Soc. Pac., San Francisco, p. 91

Arribas S., Colina L., Alonso-Herrero A., Monreal-Ibero A., García-Marín M., García-Burillo S., Rodríguez-Zaurín J., 2012, A\&A, 541, A20

Asari N. V., Cid Fernandes R., Stasińska G., Torres-Papaqui J. P., Mateus A., Sodré L., Schoenell W., Gomes J. M., 2007, MNRAS, 381, 263

Asplund M., Grevesse N., Sauval A. J., Scott P., 2009, ARA\&A, 47, 481

Baldwin J. A., Phillips M. M., Terlevich R., 1981, PASP, 93, 5

Barrera-Ballesteros J. K., Sánchez S. F., García-Lorenzo B. et al., 2015, A\&A, 579, A45

Bica E., Arimoto N., Alloin D., 1988, A\&A, 202, 8

Bruzual G., Charlot S., 2003, MNRAS, 344, 1000

Bryant P. M., Scoville N. Z., 1999, AJ, 117, 2632

Calzetti D., Kinney A. L., Storchi-Bergmann T., 1994, ApJ, 429, 582

Calzetti D., Armus L., Bohlin R. C., Kinney A. L., Koornneef J., StorchiBergmann T., 2000, ApJ, 533, 682

Cao C., Xu C. K., Domingue D. et al., 2016, ApJS, 222, 16

Chabrier G., 2003, PASP, 115, 763

Chandar R., Fall S. M., Whitmore B. C., 2015, ApJ, 810, 1

Cid Fernandes R., González Delgado R. M., 2010, MNRAS, 403, 780

Cid Fernandes R., Leão J. R. S., Lacerda R. R., 2003, MNRAS, 340, 29

Cid Fernandes R., Mateus A., Sodré L., Stasińska G., Gomes J. M., 2005, MNRAS, 358, 363

Cid Fernandes R., Stasińska G., Mateus A., Vale Asari N., 2011, MNRAS, 413,1687

Cid Fernandes R. et al., 2013, A\&A, 557, A86 (CF2013)

Cid Fernandes R. et al., 2014, A\&A, 561, A130

Colina L., Arribas S., Monreal-Ibero A., 2005, ApJ, 621, 725

Cox T. J., Jonsson P., Somerville R. S., Primack J. R., Dekel A., 2008, MNRAS, 384, 386

Crowther P. A., 2007, ARA\&A, 45, 177

Davies R. L., Medling A. M., U V., Max C. E., Sanders D., Kewley L. J., 2016, MNRAS, 458, 158

Di Matteo P., Combes F., Melchior A.-L., Semelin B., 2007, A\&A, 468, 61

Di Matteo P., Bournaud F., Martig M., Combes F., Melchior A.-L., Semelin B., 2008, A\&A, 492, 31

Dinshaw N., Evans A. S., Epps H., Scoville N. Z., Rieke M., 1999, ApJ, 525,702

Ellison S. L., Patton D. R., Simard L., McConnachie A. W., 2008, AJ, 135, 1877

Elmegreen D. M., Elmegreen B. G., Kaufman M., Sheth K., Struck C., Thomasson M., Brinks E., 2006, ApJ, 642, 158

Evans A. S. et al., 2008, ApJ, 675, L69

Filippenko A. V., 1982, PASP, 94, 715

García-Benito R. et al., 2015, A\&A, 576, A135

García-Marín M., Colina L., Arribas S., Monreal-Ibero A., 2009, A\&A, 505, 1319

Goldader J. D., Meurer G., Heckman T. M., Seibert M., Sanders D. B., Calzetti D., Steidel C. C., 2002, ApJ, 568, 651

González Delgado R. M., 2008, in Knapen J. H., Mahoney T. J., Vazdekis A., eds, ASP Conf. S. Vol. 390, Pathways Through an Eclectic Universe. Astron. Soc. Pac., San Francisco, p. 205

González Delgado R. M., Cid Fernandes R., 2010, MNRAS, 403, 797
González Delgado R. M., Cid Fernandes R., Pérez E., Pérez E., Martins L. P., Storchi-Bergmann T., Schmitt H., Heckman T., Leitherer C., 2004, ApJ, 605, 127

González Delgado R. M., Cerviño M., Martins L. P., Leitherer C., Hauschildt P. H., 2005, MNRAS, 357, 945

González Delgado R. M. et al., 2014, A\&A, 562, A47

González Delgado R. M. et al., 2015a, A\&A, 581, A103 (GD2015)

González Delgado R. M. et al., 2015b, Highlights of Spanish Astrophysics VIII. p. 220

González Delgado R. M. et al., 2016, A\&A, 590, A44

Grimes J. P., Heckman T., Hoopes C., Strickland D., Aloisi A., Meurer G., Ptak A., 2006, ApJ, 648, 310

Guo R., Hao C.-N., Xia X.-Y., Wei P., Guo X., 2016, Res. Astron. Astrophys., 16,113

Hattori T. et al., 2004, AJ, 127, 736

Hopkins P. F., Cox T. J., Hernquist L., Narayanan D., Hayward C. C., Murray N., 2013, MNRAS, 430, 1901

Iono D. et al., 2013, PASJ, 65, L7

Jarrett T. H. et al., 2013, AJ, 145, 6

Kauffmann G. et al., 2003, MNRAS, 346, 1055 (K03)

Kennicutt R. C., Jr, 1998, ARA\&A, 36, 189

Kennicutt R. C., Jr et al., 2009, ApJ, 703, 1672

Kewley L. J., Dopita M. A., 2002, ApJS, 142, 35

Kewley L. J., Heisler C. A., Dopita M. A., Lumsden S., 2001, ApJS, 132, 37 (K01)

Kewley L. J., Rupke D., Zahid H. J., Geller M. J., Barton E. J., 2010, ApJ, 721, L48

Kim D.-C. et al., 2013, ApJ, 768, 102

Knop R. A., Soifer B. T., Graham J. R., Matthews K., Sanders D. B., Scoville N. Z., 1994, AJ, 107, 920

Kreckel K. et al., 2013, ApJ, 771, 62

Krist J. E., Hook R. N., Stoehr F., 2011, in Kahan M. A., ed., Proc. SPIE Conf. Ser. Vol. 8127, Optical Modeling and Performance Predictions V. SPIE, Bellingham, p. 81270J

Larsen S. S., Brodie J. P., 2000, AJ, 120, 2938

Larson R. B., Tinsley B. M., 1978, ApJ, 219, 46

Le Borgne J.-F. et al., 2003, A\&A, 402, 433

Le Floc'h E., Charmandaris V., Laurent O., Mirabel I. F., Gallais P., Sauvage M., Vigroux L., Cesarsky C., 2002, A\&A, 391, 417

Marino R. A. et al., 2013, A\&A, 559, A114

Martins L. P., González Delgado R. M., Leitherer C., Cerviño M., Hauschildt P., 2005, MNRAS, 358, 49

Meidt S. E. et al., 2012, ApJ, 744, 17

Meidt S. E. et al., 2014, ApJ, 788, 144

Monreal-Ibero A., Arribas S., Colina L., 2006, ApJ, 637, 138

Osterbrock D. E., Ferland G. J., 2006, in Osterbrock D. E., Ferland G. J., eds, Astrophysics of Gaseous Nebulae and Active Galactic Nuclei, 2nd edn. University Science Books, Sausalito, CA, p. 72

Panter B., Jimenez R., Heavens A. F., Charlot S., 2007, MNRAS, 378, 1550

Patton D. R., Torrey P., Ellison S. L., Mendel J. T., Scudder J. M., 2013, MNRAS, 433, L59

Pérez E. et al., 2013, ApJ, 764, L1

Renaud F., Bournaud F., Kraljic K., Duc P.-A., 2014, MNRAS, 442, L33

Renaud F., Bournaud F., Duc P.-A., 2015, MNRAS, 446, 2038

Renzini A., Peng Y.-j., 2015, ApJ, 801, L29

Rich J. A., Kewley L. J., Dopita M. A., 2011, ApJ, 734, 87

Rich J. A., Torrey P., Kewley L. J., Dopita M. A., Rupke D. S. N., 2012, ApJ, 753, 5

Rich J. A., Kewley L. J., Dopita M. A., 2014, ApJ, 781, L12

Roth M. M. et al., 2005, PASP, 117, 620

Rupke D. S. N., Kewley L. J., Chien L.-H., 2010, ApJ, 723, 1255

Sánchez S. F., 2006, Astron. Nachr., 327, 850

Sánchez S. F. et al., 2012, A\&A, 538, A8

Sánchez S. F. et al., 2015, A\&A, 574, A47

Sánchez-Blázquez P. et al., 2006, MNRAS, 371, 703

Sánchez-Blázquez P., Ocvirk P., Gibson B. K., Pérez I., Peletier R. F., 2011, MNRAS, 415, 709

Sanders D. B., Mirabel I. F., 1996, ARA\&A, 34, 749 
Sanders D. B., Mazzarella J. M., Kim D.-C., Surace J. A., Soifer B. T., 2003, AJ, 126, 1607

Sirianni M. et al., 2005, PASP, 117, 1049

Surace J. A., Sanders D. B., Vacca W. D., Veilleux S., Mazzarella J. M., 1998, ApJ, 492, 116

Surace J. A., Sanders D. B., Evans A. S., 2000, ApJ, 529, 170

Teyssier R., Chapon D., Bournaud F., 2010, ApJ, 720, L149

Toomre A., Toomre J., 1972, ApJ, 178, 623

Vazdekis A., Sánchez-Blázquez P., Falcón-Barroso J., Cenarro A. J., Beasley

M. A., Cardiel N., Gorgas J., Peletier R. F., 2010, MNRAS, 404, 1639

Veilleux S., Osterbrock D. E., 1987, ApJS, 63, 295

Veilleux S., Kim D.-C., Sanders D. B., 2002, ApJS, 143, 315

Wang Z. et al., 2004, ApJS, 154, 193

Wilson C. D., Harris W. E., Longden R., Scoville N. Z., 2006, ApJ, 641, 763

\section{APPENDIX A: STAR CLUSTER PHOTOMETRY}

\section{A1 Method}

In Section 3, we have summarized the stellar population properties of the SSCs in IC $1623 \mathrm{~W}$ and NGC 6090 using the HST images from FUV to NIR. Their characteristics are summarized in Tables A1 and A2, respectively.

Here we describe in detail the methodology we have followed to perform the star cluster photometry. The clusters were detected in a combination of the HST optical images in F435W and F814W filters. We registered and resampled all the $H S T$ images to a common pixel scale, using GEOMAP/GEOTRAN IRAF tasks. Prior to the detection, we had to remove large-scale background variations in this optical image in two steps. First, it was smoothed with a $9 \times 9$ box median filter, and then, the smoothed image was subtracted from the original. The input object list for photometry was obtained by running DAOFIND task in DAOPHOT package on this background-subtracted image. This procedure is similar to that followed by Larsen \& Brodie (2000). As an additional selection criterion, only clusters with $\mathrm{S} / \mathrm{N} \geq 5$ in optical filters were considered for subsequent photometry. The detected clusters were shown in Fig. 3.

Photometry was obtained using the phot task in APРнот. It gives the fluxes of the clusters in all the filters, by specifying the aperture radius and the position and width of the sky ring for background determination. We have followed the recommendations of the reference guide to IRAF/APPHOT package (Lindsey E. Davis), selecting an aperture radius $\sim \mathrm{FWHM}_{\mathrm{PSF}}$, and $\sim 4 \times \mathrm{FWHM}_{\mathrm{PSF}}$ for the sky ring inner radius and width. The aperture photometry of sources requires the correction for the light loss due to the finite size of the aperture used, which is called the aperture correction. The best way to determine aperture corrections is from real point sources (stars) on the images. However, isolated bright stars to obtain a meaningful correction were only present in optical images, but nor in UV neither in NIR. Thus, aperture corrections were determined from analytically generated point sources using TINY TIM software (Krist, Hook \& Stoehr 2011).

We have converted these fluxes into Space Telescope Science Institute magnitude system (STMAG), using equation 6 in Sirianni et al. (2005). The errors have been calculated through error propagation:

$\Delta F=\sqrt{F+n_{\text {aper }} \times\left(s k y+\sigma_{\mathrm{RO}}^{2}\right) \times\left(1+\frac{n_{\text {aper }}}{n_{\text {sky }}}\right)}$,

where $F$ is the star cluster flux within the aperture, $n_{\text {aper }}$ is the number of pixels in the aperture, sky is the sky background value per pixel, measured in the sky annulus around the cluster, $n_{\text {sky }}$ is the number of pixels in the background annulus and $\sigma_{\mathrm{RO}}$ is the readout noise of the CCD. Errors in the corresponding magnitudes were computed from equation (A1) using $\Delta m=2.5 \log e \times \Delta F / F$.

\section{A2 Cluster ages}

To estimate cluster properties, we have compared the colours and magnitudes of IC 1623 and NGC 6090 clusters with those of Charlot \& Bruzual SSPs models (2007, unpublished) in a range of ages between $1 \mathrm{Myr}$ and $13 \mathrm{Gyr}$. These models are similar to those in Bruzual \& Charlot (2003), but replacing STELIB (Le Borgne et al. 2003) by a combination of the MILES (Sánchez-Blázquez et al. 2006) and GRANADA (Martins et al. 2005) libraries. Chabrier IMF and Padova 1994 evolutionary tracks were used. Model colours have been computed using STSDAS.SYNPHOT software. Using the Calzetti et al. (2000) law, we have computed also the colours of the models reddened by up to $A_{V}=6 \mathrm{mag}$. Despite recent improvements

Table A1. Summary of HST data for IC 1623.

\begin{tabular}{lccccrc}
\hline \multicolumn{1}{c}{ Filter } & Instrument & $\begin{array}{c}\text { Detector/ } \\
\text { Camera }\end{array}$ & $\begin{array}{c}\text { Plate scale } \\
\left(\operatorname{arcsec~pixel}{ }^{-1}\right)\end{array}$ & $\begin{array}{c}\text { Observation } \\
\text { date }\end{array}$ & $\begin{array}{c}t_{\exp } \\
(\mathrm{s})\end{array}$ & $\begin{array}{c}\text { Proposal ID, } \\
\text { PI }\end{array}$ \\
\hline$F 25 S R F 2$ & STIS & FUV-MAMA & 0.025 & $2000-12-25$ & 2371.0 & 8201 , G. Meurer \\
$F 25 Q T Z$ & STIS & NUV-MAMA & 0.025 & $2000-12-25$ & 750.0 & 8201 , G. Meurer \\
$F 435 W$ & ACS & WFC & 0.05 & $2006-07-12$ & 1260.0 & 10592, A. Evans \\
$F 814 W$ & ACS & WFC & 0.05 & $2006-07-12$ & 720.0 & 10592 , A. Evans \\
$F 110 W$ & NICMOS & NIC2 & 0.075 & $1998-08-03$ & 223.8 & 7219 , N. Scoville \\
$F 160 W$ & NICMOS & NIC2 & 0.075 & $1998-08-03$ & 223.8 & 7219 , N. Scoville \\
\hline
\end{tabular}

Table A2. Summary of HST data for NGC 6090.

\begin{tabular}{lccccrc}
\hline Filter & Instrument & $\begin{array}{c}\text { Detector/ } \\
\text { Camera }\end{array}$ & $\begin{array}{c}\text { Plate scale } \\
\left(\operatorname{arcsec} \text { pixel }^{-1}\right)\end{array}$ & $\begin{array}{c}\text { Observation } \\
\text { Date }\end{array}$ & $\begin{array}{c}t_{\exp } \\
(\mathrm{s})\end{array}$ & $\begin{array}{c}\text { Proposal ID, } \\
\text { PI }\end{array}$ \\
\hline$F 140 L P$ & ACS & SBC & 0.025 & $2008-08-09$ & 2648.0 & 11110 , S. McCandliss \\
$F 330 W$ & ACS & HRC & 0.025 & $2005-11-12$ & 800.0 & 10575 , G.Ostlin \\
$F 435 W$ & ACS & WFC & 0.05 & $2005-09-18$ & 1380.0 & 10592 , A. Evans \\
$F 814 W$ & ACS & WFC & 0.05 & $2005-09-18$ & 800.0 & 10592 , A. Evans \\
$F 110 W$ & NICMOS & NIC2 & 0.075 & $1997-11-10$ & 383.6 & 7219 , N. Scoville \\
$F 160 W$ & NICMOS & NIC2 & 0.075 & $2003-12-01$ & 599.4 & 9726 , R. Maiolino \\
\hline
\end{tabular}



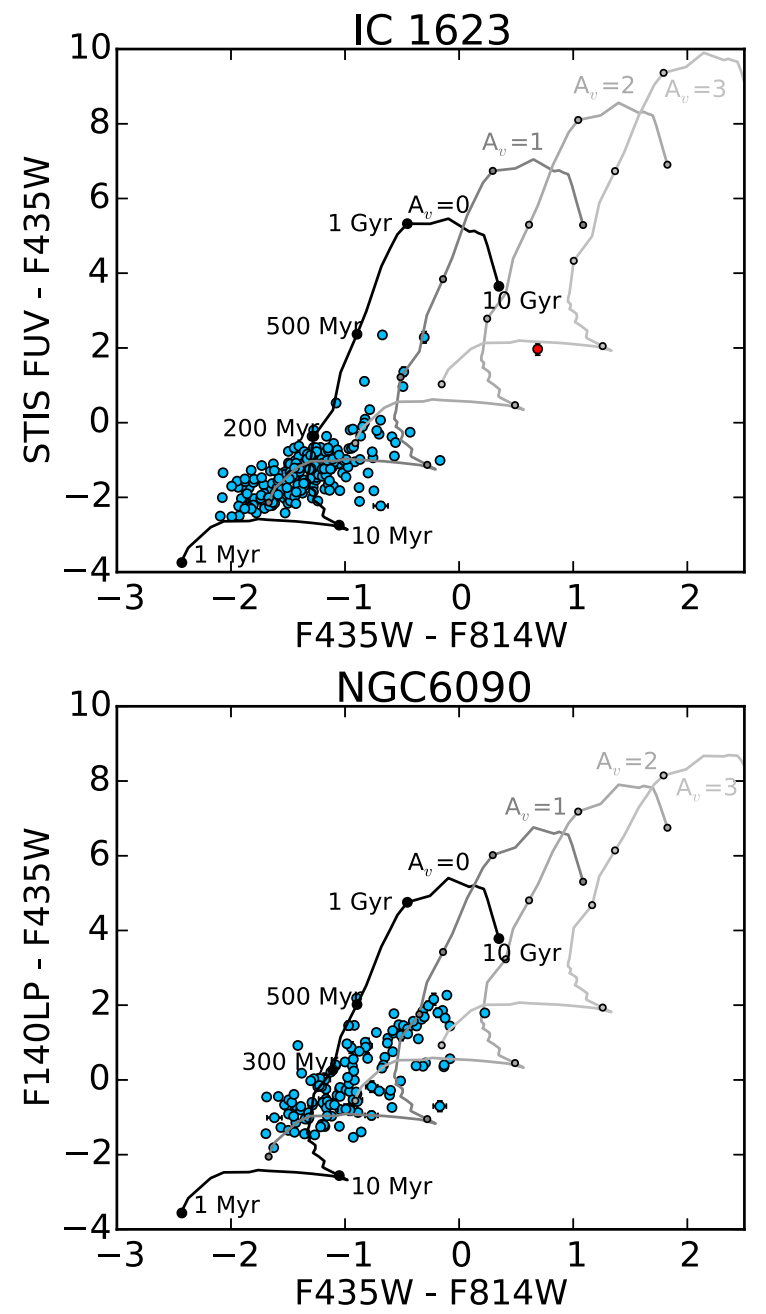

Figure A1. FUV $-F 435 W$ versus $F 435 W-F 814 W$ diagram for IC 1623 clusters (top panel) and NGC 6090 clusters (bottom panel). The colour coding is the same as in Fig. 3, but only those clusters with SNR $>5$ in FUV, $F 435 \mathrm{~W}$ and $F 814 \mathrm{~W}$ filters are included in the plots. The black solid line is the path described by SSP from $1 \mathrm{Myr}$ to $13 \mathrm{Gyr}, \mathrm{Z}_{\odot}$ and $A_{V}=$ 0 mag. The grey-scale lines are the paths for the same models reddened by 1-3 mag, with the lighter shades tracing the more extincted models. For both systems, most clusters are consistent with ages younger than 200-300 Myr, assuming very low to almost no foreground extinction. If affected by some extinction, 0.5-1.0 mag, they could be even younger than $10 \mathrm{Myr}$.

in modelling techniques, it is very difficult from photometry to estimate metallicity on individual clusters. From the spectroscopy, we found that the average value for the metallicity in these systems is $\sim 0.6 \mathrm{Z}_{\odot}$ (see Section 4.6). Taking this into account, we have used solar metallicity SSP models $\left(1 \mathrm{Z}_{\odot}\right)$ in this analysis. However, we have checked that $0.4 \mathrm{Z}_{\odot}$ models predict similar results.

In Fig. A1, we show the FUV $-F 435 W$ versus $F 435 W-F 814 W$ diagram for IC 1623 (top panel) and NGC 6090 (bottom panel), which is the best to break the age- $A_{V}$ degeneracy. The colour coding is the same as in Fig. 3, but only those clusters with $\mathrm{SNR} \geq 5$ in FUV, $F 435 \mathrm{~W}$ and $F 814 \mathrm{~W}$ filters are included in the plots. The black solid line is the path described by SSP models from $1 \mathrm{Myr}$ to $13 \mathrm{Gyr}, \mathrm{Z}_{\odot}$ and $A_{V}=0 \mathrm{mag}$. The grey-scale lines are the paths for the same models reddened by 1-3 mag, with the lighter shades tracing the more reddened models.
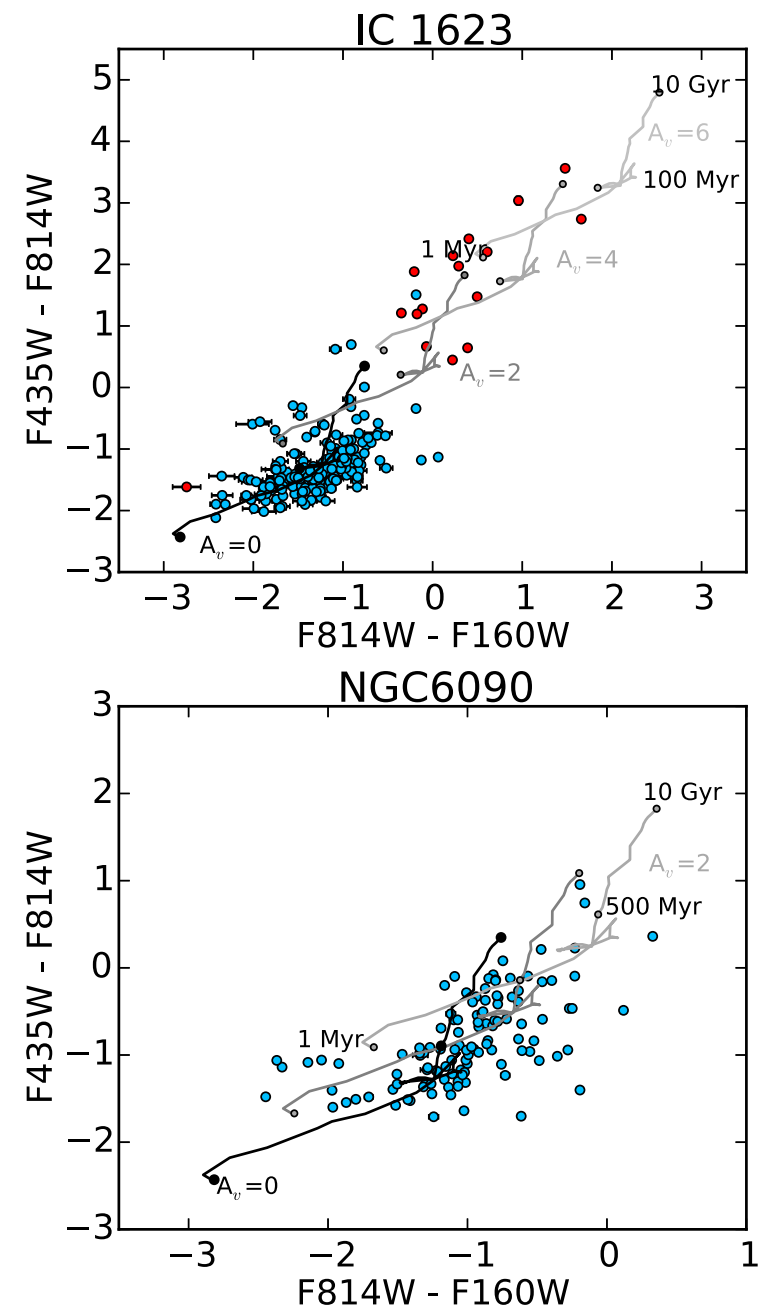

Figure A2. $F 435 W-F 814 W$ versus $F 814 W-F 160 W$ diagram for IC 1623 clusters (top panel) and NGC 6090 (bottom panel). Only clusters with SNR $>5$ in $F 435 W, F 814 W$ and $F 160 W$ filters are shown. The black solid line is the path described by SSPs from $1 \mathrm{Myr}$ to $13 \mathrm{Gyr}, \mathrm{Z}_{\odot}$ and $A_{V}=$ 0 mag. The grey-scale lines are the paths for the same models but reddened by 1,2, 4 and 6 mag.

Comparing the position of the clusters in colour-colour diagrams with respect to the model positions, we can estimate the range of cluster ages. The main results are summarized in Section 3.

\section{A3 Cluster extinction}

Fig. A2 shows the $F 435 W-F 814 W$ versus $F 814 W-F 160 W$ diagram for IC 1623 (top panel) and NGC 6090 (bottom panel). This diagram allows us to estimate the range of extinction affecting the clusters that are obscured in the FUV. Only clusters with $\mathrm{SNR} \geq 5$ in $F 435 W, F 814 W$ and $F 160 W$ filters are shown. Again, the black solid line is the path described by SSPs from $1 \mathrm{Myr}$ to $13 \mathrm{Gyr}, \mathrm{Z}_{\odot}$ and $A_{V}=0$ mag. The grey-scale lines are the paths for the same models reddened by up to $6 \mathrm{mag}$, with the lighter shades tracing the more extincted models.

We note that while in NGC 6090, the difference in the values of extinction between the two progenitors is relatively small, $\sim 1$ mag, for IC 1623, the contrast between both progenitors is much higher, possibly up to 4-6 mag. 

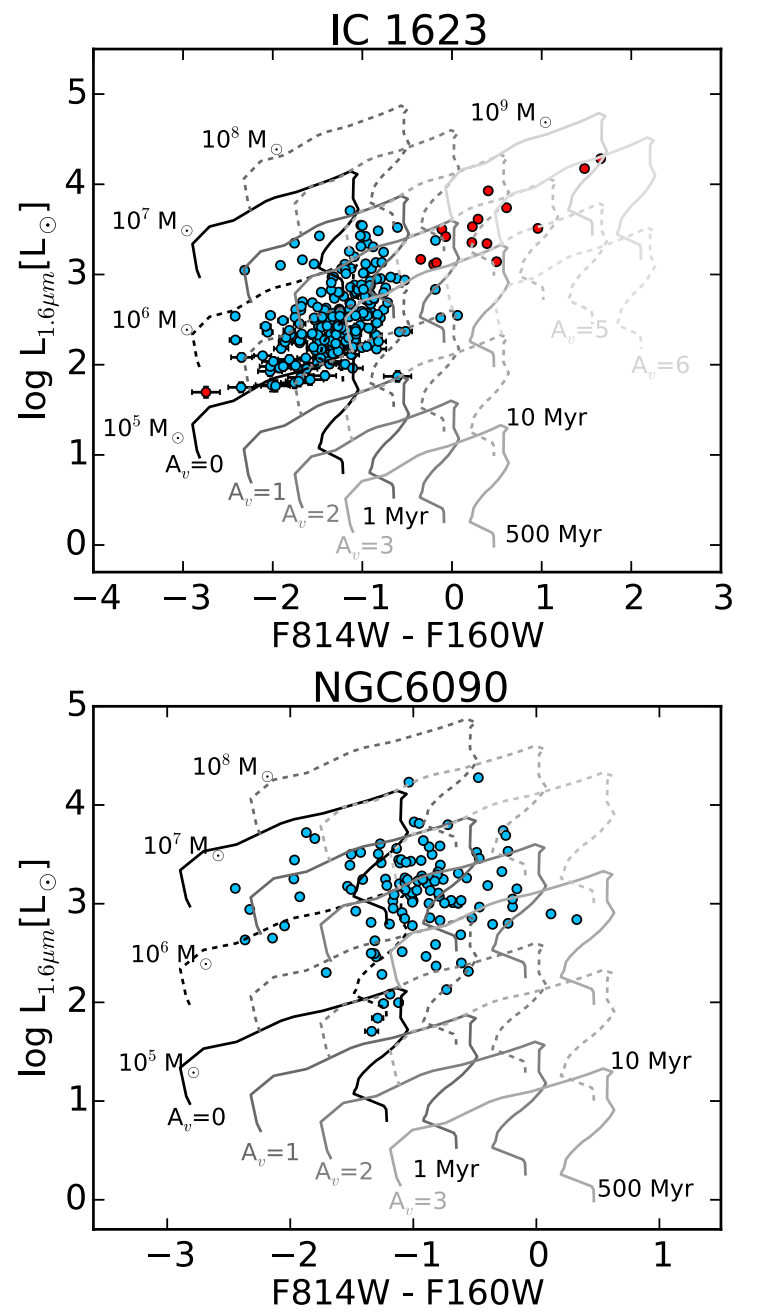

Figure A3. Absolute NIR $1.6 \mu \mathrm{m}$ magnitude versus $F 814 \mathrm{~W}-\mathrm{F} 160 \mathrm{~W}$ diagram for IC1623 clusters (top panel) and NGC 6090 clusters (bottom panel). Only clusters with SNR $>5$ in $F 435 W, F 814 W$ and $F 160 W$ filters are shown. The colours of the clusters are the same as in Fig. 3. The paths from models of $10^{5}, 10^{6}, 10^{7}, 10^{8}$ and $10^{9} \mathrm{M}_{\odot}$ have been shown as solid and dashed lines. The models have been cut between 1 and $500 \mathrm{Myr}$, as we know from previous diagrams that the clusters are not older than $500 \mathrm{Myr}$ in these two early-stage merger LIRGs. The grey-scale coding represents the variation in stellar extinction as in previous figures.

\section{A4 Cluster mass}

We found that NICMOS $1.6 \mu \mathrm{m}$ band is the most sensitive to mass variations. We estimate the mass range of the star clusters by comparing their $1.6 \mu \mathrm{m}$ absolute magnitude with the absolute magnitude of SSPs models of masses ranging from $10^{5}$ to $10^{9} \mathrm{M}_{\odot}$. The results are shown in Figure A3 for IC 1623 (top panel) and NGC 6090 (bottom panel). This is a NIR $1.6 \mu \mathrm{m}$ absolute magnitude versus $F 814 \mathrm{~W}-F 160 \mathrm{~W}$ diagram. The paths from models of $10^{5}$, $10^{6}, 10^{7}, 10^{8}$ and $10^{9} \mathrm{M}_{\odot}$ are shown as solid and dashed lines. The models have been cut between 1 and $500 \mathrm{Myr}$, as we know from the previous section that these are the older ages our clusters can have.

We have performed a rough estimate of the total mass in clusters in these systems by assigning a certain average mass for the clusters in each luminosity $L_{1.6 \mu \mathrm{m}}$ range. In particular, we have separated the clusters in four possible luminosity ranges: from $0.9<\log L_{1.6 \mu \mathrm{m}}<1.5$, we assign a mass of $\sim 10^{5} \mathrm{M}_{\odot}$; if $1.5<\log$ $L_{1.6 \mu \mathrm{m}}<2.65$, then $\sim 10^{6} \mathrm{M}_{\odot}$; if $2.65<\log L_{1.6 \mu \mathrm{m}}<3.5$, then $\sim 10^{7} \mathrm{M}_{\odot}$, and above $\log L_{1.6 \mu \mathrm{m}}>3.5$, we assign $\sim 10^{8} \mathrm{M}_{\odot}$. In the case of IC $1623 \mathrm{~W}$, we have $0,143,62$ and 1 clusters in each range, leading to a clusters mass $\mathrm{M}_{\mathrm{IC} 1623 W}^{\text {clus }} \sim 9 \times 10^{8} \mathrm{M}_{\odot}$. In NGC 6090, we have 0,19, 79, 19 clusters in each luminosity range, leading to $\mathrm{M}_{\mathrm{NGC6090}}^{\text {clus }} \sim 2 \times 10^{9} \mathrm{M}_{\odot}$.

\section{A5 Cluster detection limits}

A major question to be addressed with star cluster photometry is to know if older/more reddened clusters could have been detected. As the star cluster analysis was a complementary goal, we did not make a proper completeness study as in Davies et al. (2016), where synthetic clusters of different ages/optical depths are introduced in the cluster detection image to quantify which percentage of them could be recovered. However, we followed a more simplistic approach to estimate which clusters could be, in principle, detected, and which not. Using the SSP models of $10 \mathrm{Myr}, 100 \mathrm{Myr}, 200 \mathrm{Myr}, 300 \mathrm{Myr}$, $500 \mathrm{Myr}$ and $1 \mathrm{Gyr}$, and the transmission curves from $\mathrm{F} 435 \mathrm{~W}$ and $F 814 W$ filters, we compute the 435 and 814 mag for clusters of different masses $\left(10^{5}, 10^{6}, 10^{7}\right.$ and $\left.10^{8} \mathrm{M}_{\odot}\right)$ and affected by extinctions of $0,1,2,4,6$ and $10 \mathrm{mag}$. These magnitudes are compared with the 435 and 814 limiting mag expected for point sources (most clusters are not resolved) with $\mathrm{SNR}=5$, obtained from figs 10.2 and 10.32 of the ACS Instrument Handbook, given the exposure times of the $H S T$ observations used in this paper $\left(\sim 10^{3} \mathrm{~s}\right)$.

The less massive the cluster, the more difficult it is to detect it as it ages/is more reddened. In IC 1623 , clusters of $10^{5} \mathrm{M}_{\odot}$ up to $300 \mathrm{Myr}$ can be detected for no extinction; for $A_{V}=1 \mathrm{mag}$, clusters older than $100 \mathrm{Myr}$ cannot be detected, and for $A_{V}=2-4$ mag, only clusters of $10 \mathrm{Myr}$ can be detected. In NGC 6090, whose distance is larger than IC 1623 one, only $10^{5} \mathrm{M}_{\odot}$ clusters up to $100 \mathrm{Myr}$ can be detected for no extinction, while for $A_{V}=1-2 \mathrm{mag}$, only $10 \mathrm{Myr}$ clusters can be detected.

From our observations, we found that most clusters in IC 1623 and NGC 6090 have masses between $10^{6}$ and $10^{7} \mathrm{M}_{\odot}$. In principle, $10^{6} \mathrm{M}_{\odot}$ clusters up to $1 \mathrm{Gyr}$ (in IC 1623) and $500 \mathrm{Myr}$ (NGC 6090) could be detected up to extinction levels of $A_{V}=2$ mag. For $A_{V}=4 \mathrm{mag}$, only clusters up to 300 (IC 1623) and $100 \mathrm{Myr}$ (NGC 6090) can be detected, and for $A_{V}=6 \mathrm{mag}$, only $10 \mathrm{Myr}$ clusters can be detected. Above that extinction level, we cannot detect clusters anymore.

The age/extinction limits reach further for the more massive clusters. For example, $10^{7}-10^{8} \mathrm{M}_{\odot}$ clusters in IC $1623 \mathrm{E}$ can be detected up to ages of $1 \mathrm{Gyr}$, and $A_{V}=6$ mag.

Because point sources are much harder to detect on top of the diffuse/changing galaxy background than when isolated (which is what the HST manual assumes), we acknowledge that our detection limits are in fact upper limits, as they consider cluster detection under optimal conditions. More realistically, the detection limits become more restrictive depending on the galaxy background.

From this analysis, we conclude that star clusters photometry only allows us to trace the recent SFH of these mergers, $<500 \mathrm{Myr}-$ $1 \mathrm{Gyr}$ (at best, for no extinction), but not in previous epochs. So if older star formation occurred, complementary methods will be necessary to detect it as stellar population synthesis. Therefore, star cluster studies are preferably indicated in early-stage mergers where most of the merger-induced star formation is recent, or in more advanced mergers if we are only interested in the youngest populations formed. 
APPENDIX B: ALTERNATIVE RADIAL PROFILES FOR NGC $6090 \mathrm{SW}$

As commented in Section 2.4, the exact position of NGC 6090 SW nucleus is not clear. In the radial profiles shown in previous sections, we considered as the nucleus the luminosity peak in the IFS continuum, marked as a black dot in the maps. However, there exists the possibility that the real nucleus is the bright knot located to the north-west of this progenitor, which is indicated with a $\mathrm{km} \mathrm{s}^{-1}$ bol in the maps. As a sanity check, we have repeated the radial profiles of the stellar population properties taking the knot posi- tion as origin. The only property for which the radial profile changes is the metallicity. The trend is the same: Metallicity grows with distance from the core, but the gradient is steeper from the knot than from our nucleus. From the knot, it goes from $\left\langle\log Z_{\star} / \mathrm{Z}_{\odot}\right\rangle_{\mathrm{m}}=-0.6$ to -0.15 , while for our nucleus, it goes from -0.4 to -0.25 . For the other properties, the profiles are very similar and the differences are not significant.

This paper has been typeset from a $\mathrm{T}_{\mathrm{E}} \mathrm{X} / \mathrm{LAT} \mathrm{E} \mathrm{X}$ file prepared by the author. 